\title{
Transverse operator method for wakefields in a rectangular dielectric loaded accelerating structure
}

\author{
S. S. Baturin, ${ }^{1, *}$ I. L. Sheinman, ${ }^{1}$ A. M. Altmark, ${ }^{1}$ and A. D. Kanareykin ${ }^{1,2}$ \\ ${ }^{1}$ St. Petersburg State Electrotechnical University, St. Petersburg, Russia \\ ${ }^{2}$ Euclid Techlabs, LLC, 5900 Harper Road, Solon, Ohio 44139, USA
}

(Received 12 February 2013; published 16 May 2013)

\begin{abstract}
Cherenkov radiation generated by a relativistic electron bunch in a rectangular dielectric-loaded waveguide is analyzed under the assumption that the dielectric layers are inhomogeneous normal to the beam path. We propose a method that uses eigenfunctions of the transverse operator applied to develop a rigorous full solution for the wakefields that are generated. The dispersion equation for the structure is derived and the wakefield analysis is carried out. The formalism developed here allows the direct solution of the inhomogeneous system of Maxwell equations, an alternative analytic approach to the analysis of wakefields in contrast to the previously used impedance method for rectangular structure analysis. The formalism described here was successfully applied to the analysis of rectangular dielectric-lined structures that have been recently beam tested at the Argonne (ANL/AWA) and Brookhaven (BNL/ATF) accelerator facilities.
\end{abstract}

DOI: 10.1103/PhysRevSTAB.16.051302

PACS numbers: 41.75.Lx, 42.82.Et, 84.40.- $\mathrm{x}$

\section{INTRODUCTION}

Future high energy charged particle (electron and ion) accelerators will require new technologies to ensure expansion of the energy and intensity frontiers. Future linear colliders will need to be based on new methods since foreseeable normal and superconducting technologies are unavoidably limited in accelerating gradient. Progress in compact x-ray source design based on free electron lasers also requires high gradient nonconventional linear accelerators to be developed.

Some of the most promising new methods of charged particle acceleration are laser-plasma [1,2], beam-driven plasma [2,3], and dielectric based accelerators [4-7]. All the advantages and limitations of these schemes as well as their current status in simulations and experimental results can be found in the references. In this paper we focus on dielectric based wakefield acceleration technology (DWA) as one of the most promising new technologies for next generation linear colliders [8] and future x-ray free-electron lasers (FELs)[9].

In this paper we consider dielectric-lined structures that are excited by a high intensity relativistic electron beam to generate high power $X$-band, mm-wave, or $\mathrm{THz}$ radiation [7-9]. This rf pulse is used to drive another dielectric or conventional accelerating structure (e.g. the CLIC project power extraction structure $[10,11])$ or accelerate a second electron beam in the same structure. This method of electromagnetic wave generation avoids the need to develop

\footnotetext{
*s.s.baturin@gmail.com

Published by the American Physical Society under the terms of the Creative Commons Attribution 3.0 License. Further distribution of this work must maintain attribution to the author(s) and the published article's title, journal citation, and DOI.
}

more traditional rf sources and components that are able to sustain and transmit GW-level microwave or $\mathrm{THz}$ power into the structure [5-7].

A dielectric-loaded accelerating (DLA) or power extraction structure is a dielectric waveguide with an axial vacuum channel for beam propagation and which is surrounded by a conducting metal wall [4-6]. Recent experiments have shown that dielectric based structures can sustain accelerating gradients in excess of $100 \mathrm{MV} / \mathrm{m}$ [12] and $\mathrm{GV} / \mathrm{m}$ [6] in the upper $\mathrm{GHz}$ and $\mathrm{THz}$ frequency ranges, respectively.

For example, a high current (up to $100 \mathrm{nC}$ ) short (1-2 mm) relatively low energy (15-100 MeV) electron bunch in this type of a structure can generate Cherenkov wakefields with the longitudinal field magnitude up to $100-300 \mathrm{MV} / \mathrm{m}$ in the $X-\mathrm{Ka}$ band frequency ranges [12]. A $3 \mathrm{nC}$ charge and $30 \mu \mathrm{m}$ long bunch of the $23 \mathrm{GeV}$ SLAC/ FACET accelerator generates $1-10 \mathrm{GV} / \mathrm{m}$ wakefields in the $\mathrm{THz}$ range $[6,13]$. These wakefields are used for accelerating a less intense but high energy electron bunch propagating behind the drive bunch at a distance corresponding to the accelerating phase of the $E_{z}$ field [4,5,14]. Dielectric based structures provide, in addition to the high accelerating gradient, the possibility of controlling the frequency spectrum of the structure by introducing nonlinear dielectrics (ferroelectrics) [15,16]. There is also the potential of using new manufactured materials (such as diamond and sapphire) with unique dielectric strength and thermoconductive properties [17-19].

As a rule, the cylindrical geometry usually proposed for dielectric based structures is required for attaining the highest accelerating gradients as well as for providing the maximal possible shunt impedance of the structure $[4,5]$. Results of analytic mode analyses of cylindrical dielectric accelerating structures are found in [20,21]. 
Since 1988 when the first proof of principle experimental results were published [4], studies of cylindrical dielectric structures have been presented in numerous publications (see [5-9,11-16]).

At the same time, the dielectric-lined planar or rectangular cross section geometries are also being considered; a minor reduction in performance is acceptable in view of the technological difficulties of fabricating cylindrical structures (related to the stringent tolerance requirements for geometrical parameters and uniformity of the permittivity of the dielectric [3]). Rectangular structures can be used for measurements on new dielectric materials effective in sustaining high acceleration rates and tolerant of pulsed heating of the structure [4]. Planar structures are also being studied (along with cylindrical geometries) for generating terahertz radiation in the frequency range $0.5-1.0 \mathrm{THz}$ [4] and for energy spread reduction ("silencer") systems for FEL linacs [22].

Another advantage of using a planar geometry is related to the drive beam stability problem for DWAs. The longitudinal electric field magnitude of the wakefield is inversely proportional to the square of the vacuum channel radius; therefore it is critically important to employ small aperture structures. At the same time, reducing the vacuum channel radius increases the severity of beam breakup (BBU) effects $[5,7,23]$. Planar DWA structures can provide a reasonable compromise between the reduced accelerating gradient and the required beam transverse stability [23]. The planar option has the additional advantage that a flat electron beam can be generated directly in photoinjectors [24].

Theoretical analyses of dielectric accelerating structures with rectangular geometries have been reported in a number of publications [23,25-31]. To determine the amplitudes of individual Cherenkov radiation modes excited in a rectangular waveguide with dielectric slabs, the impedance matching technique was used in the earlier papers $[23,25,26]$. When that formalism is used instead of the direct solution of the nonhomogeneous system of Maxwell equations (the standard analytic approach in analysis of wakefields in cylindrical structures [20,21]), the amplitudes of the wakefields needs to be expressed in terms of the shunt impedance (or integrated loss factor) for each mode of the structure $[23,25,26]$. The approach involves certain approximations, while the direct solution of the inhomogeneous system of Maxwell's equations without intermediate approximations is always preferable for analyzing the wakefield generation problem in DWA structures. In this paper, a rigorous direct solution was found for the wakefields in an arbitrary rectangular dielectric-lined waveguide.

In this paper, we applied a transverse operator method that was used previously as an analytical algorithm for wave propagation of modes in a waveguide with complex loading, where the rectangular waveguide is partially filled with dielectric, ferrite, ferroelectric, or any type of anisotropy is present (chiral loading, for example) [28-34]. Some specific version of transverse operator method called the mode basis method was developed and applied to the analysis of wave propagation in conical antennas with dielectric filling $[35,36]$.

The method of the first order transverse operator has been applied to waveguide problems in [32-34]. The generalized orthogonality relations between the longitudinal magnetic and longitudinal electric modes have been derived in $[28,29]$. However, the bilinear form introduced in [28,29] is not the scalar product, and requires justification of the use of this relation for orthogonality between the components of the electric and magnetic field vectors. In [30], the analysis was performed on the basis of the second order differential equations for the transverse field components (direct solution of the Maxwell equations) of a two-channel rectangular structure with dielectric-lined waveguides. This structure design was proposed for increasing the transformer ratio from the drive beam to the beam being accelerated. However, some of the mathematical aspects of the analysis [28-30] have not been rigorously verified and need additional studies. To be specific, there is a problem of sign inversion of the weight function in the definition of the norm, and the completeness of the set of functions over which the expansion was performed was not demonstrated.

Our approach to this problem is the use of a modified transverse operator method. It should be noted that in contrast to the analysis of [28-34], we consider the Sturm-Liouville second order operator with an alternating sign weight function. It will be shown below that the integral relation derived in [28,29] is a consequence of the bi-orthogonality of the eigenfunctions as well as the similarity of the operator to a self-adjoint operator. This approach makes it possible to obtain a complete analytic solution for the eigenmodes of this system, and thus, to solve in the most general form the problem of Cherenkov wakefield generation in a rectangular accelerating structure with a composite dielectric loading.

Finally, (i) the proposed method does not require any assumptions on the form of the beam's Coulomb field (in contrast to the impedance method $[25,26]$, where it is often assumed that the field of the beam is essentially flat, an assumption that is valid only at ultrarelativistic energies); (ii) we also did not use the mode decomposition for the problem formulation and its subsequent solution; the mode series obtained as a solution emerges in a natural way as a result of solving the problem; (iii) as a consequence of (ii), and the use of the transverse operator formalism the series of modes of the solution is uniformly convergent. This is not obvious for the solution obtained by the impedance method [25,26] and requires additional analysis and proof.

\section{THEORETICAL ANALYSIS}

We consider the waveguide shown in Fig. 1. We start from the Maxwell system in CGS units (1)-(4), with the additional constitutive relations: 


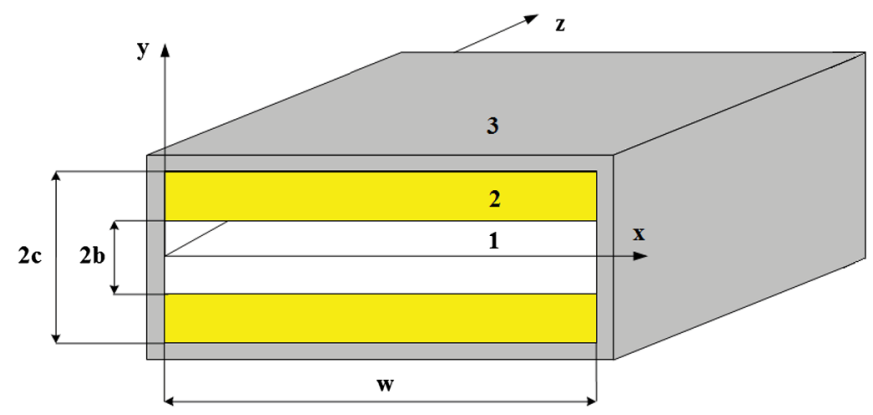

FIG. 1. A rectangular dielectric-loaded accelerating structure; (1) vacuum, (2) dielectric, (3) conducting walls.

$$
\begin{gathered}
\nabla \times \mathbf{E}=-\frac{\partial \mathbf{B}}{c \partial t} \\
\nabla \times \mathbf{H}=\frac{\partial \mathbf{D}}{c \partial t}-\frac{e n \mathbf{V}}{c} ; \\
\nabla \cdot \mathbf{B}=0 \\
\nabla \cdot \mathbf{D}=-e n \\
\mathbf{B}=\mu \mathbf{H} ; \quad \mathbf{D}=\varepsilon \mathbf{E},
\end{gathered}
$$

where $\mathbf{E}, \mathbf{H}$ are the electric and magnetic field vectors respectively; $\mathbf{D}, \mathbf{B}$ are the electric and magnetic flux vectors respectively; $\mathbf{V}$-average speed of the bunch; $n$-particle concentration of the bunch; $e$-electron charge; $t$-time.

The electron bunch is moving in the vacuum channel along the waveguide axis. The wakefield is generated by the bunch when the Cherenkov condition $v / c=$ $\beta>\varepsilon^{-1 / 2}$ is satisfied. Here $c$ is the speed of light and $v$ the speed of the moving particle (or bunch).

For a point charge $q$, moving along waveguide's $z$ axis the charge distribution is given by

$$
n(x, y, z, t)=4 \pi N \delta(z-v t) \delta\left(x-x_{0}\right) \delta\left(y-y_{0}\right),
$$

where $x, y$ are transverse coordinates, $z$ is the longitudinal coordinate, and $x_{0}, y_{0}$ are the transverse coordinates of the beam, $N$ number of electrons in the bunch, bunch charge $q=-e N$.

We consider a rectangular waveguide with symmetric dielectric layers placed parallel to the OZX plane and separated by a vacuum gap. In this case the dielectric is inhomogeneous along the $y$ axis, so that $\varepsilon=\varepsilon(y), \mu=$ $\mu(y)$. Next we evaluate the Maxwell system (1)-(4) with (5) for this case. From (4) we obtain

$$
\nabla \cdot \mathbf{E}=-\left(\frac{E_{y}}{\varepsilon} \frac{\partial \varepsilon}{\partial y}+\frac{e n}{\varepsilon}\right)
$$

applying the curl operator to (1) and using (7), we get

$$
\begin{gathered}
\frac{\partial^{2} \mathbf{E}}{\partial z^{2}}+\Delta_{x, y} \mathbf{E}+\nabla\left[\frac{E_{y}}{\varepsilon} \frac{\partial \varepsilon}{\partial y}\right]-\varepsilon \mu \frac{\partial^{2} \mathbf{E}}{c^{2} \partial t^{2}} \\
=-e\left[\nabla\left(\frac{n}{\varepsilon}\right)+\mu \beta \frac{\partial n}{c \partial t} \frac{\mathbf{V}}{|\mathbf{V}|}\right]+\frac{\partial \boldsymbol{\Lambda}}{c \partial t}
\end{gathered}
$$

where we define

$$
\boldsymbol{\Lambda} \equiv\left(\begin{array}{c}
H_{z} \frac{\partial \mu}{\partial y} \\
0 \\
-H_{x} \frac{\partial \mu}{\partial y}
\end{array}\right),
$$

and

$$
\Delta_{x, y} \equiv \frac{\partial^{2}}{\partial x^{2}}+\frac{\partial^{2}}{\partial y^{2}}
$$

By inspection of Eq. (8), only the equation for the $E_{y}$ component does not include additional terms depending on other field components. In other words, only the $E_{y}$ equation is independent.

The equation for the $E_{y}$ component of the electric field given by (8) is

$$
\frac{\partial^{2} E_{y}}{\partial z^{2}}+\Delta_{x, y} E_{y}+\frac{\partial}{\partial y}\left[\frac{E_{y}}{\varepsilon} \frac{\partial \varepsilon}{\partial y}\right]-\varepsilon \mu \frac{\partial^{2} E_{y}}{c^{2} \partial t^{2}}=-e \frac{\partial}{\partial y}\left[\frac{n}{\varepsilon}\right] .
$$

The terms of the operator on the left side of the equation containing only derivatives with respect to $y$ can be simplified as

$$
\frac{\partial^{2} E_{y}}{\partial y^{2}}+\frac{\partial}{\partial y}\left[\frac{E_{y}}{\varepsilon} \frac{\partial \varepsilon}{\partial y}\right]=\frac{\partial}{\partial y}\left[\frac{1}{\varepsilon} \frac{\partial}{\partial y}\left[\varepsilon E_{y}\right]\right] .
$$

We introduce the new variable $\zeta=z-v t$ and we will look for a solution in the form $E_{y}(x, y, z, t)=$ $E_{y}(x, y, z-v t)=E_{y}(x, y, \zeta)$. The equation for $E_{y}$ then becomes

$\frac{\partial^{2} E_{y}}{\partial \zeta^{2}}\left(1-\varepsilon \mu \beta^{2}\right)+\frac{\partial^{2} E_{y}}{\partial x^{2}}+\frac{\partial^{2} E_{y}}{\partial y^{2}}+\frac{\partial}{\partial y}\left(\frac{E_{y}}{\varepsilon} \frac{\partial \varepsilon}{\partial y}\right)=\frac{-e}{\varepsilon_{0}} \frac{\partial}{\partial y}\left(\frac{n}{\varepsilon}\right)$.

We introduce the operator

$$
\hat{T}_{E} E_{y}=\frac{1}{\left(1-\varepsilon \mu \beta^{2}\right)}\left[\frac{\partial^{2} E_{y}}{\partial x^{2}}+\frac{\partial}{\partial y}\left(\frac{1}{\varepsilon} \frac{\partial}{\partial y}\left[\varepsilon E_{y}\right]\right)\right] \text {. }
$$

We can then rewrite (13) as

$$
\frac{\partial^{2} E_{y}}{\partial \zeta^{2}}+\hat{T}_{E} E_{y}=\frac{-e}{\varepsilon_{0}\left(1-\varepsilon \mu \beta^{2}\right)} \frac{\partial}{\partial y}\left(\frac{n}{\varepsilon}\right) .
$$

Following the same steps for the magnetic part of the Maxwell system we obtain

$$
\Delta \mathbf{H}+\nabla\left[\frac{\mathbf{H} \cdot \nabla \mu}{\mu}\right]=-\frac{\partial(\nabla \times \mathbf{E})}{c \partial t}-\frac{\partial \Xi}{c \partial t}+\frac{e}{c} \nabla \times(n \mathbf{V}),
$$


where

$$
\Xi \equiv\left(\begin{array}{c}
E_{z} \frac{\partial \varepsilon}{\partial y} \\
0 \\
-E_{x} \frac{\partial \varepsilon}{\partial y}
\end{array}\right) .
$$

The velocity vector $\mathbf{V}$ has only a $z$ component, so

$$
\nabla \times(n \mathbf{V})=v\left(\begin{array}{c}
\frac{\partial n}{\partial y} \\
-\frac{\partial n}{\partial x} \\
0
\end{array}\right)
$$

We should mention here that for the magnetic vector the situation is similar to the electric case in that the only equation that does not depend on other field components is the one for $H_{y}$.

Thus combining (16)-(18), we can write the equation for the $H_{y}$ component as

$$
\Delta H_{y}+\frac{\partial}{\partial y}\left[\frac{H_{y}}{\mu} \frac{\partial \mu}{\partial y}\right]-\varepsilon \mu \frac{\partial^{2} H_{y}}{c^{2} \partial t^{2}}=-e \beta \frac{\partial n}{\partial x} .
$$

As before, we seek a solution in the form $H_{y}(x, y, z, t)=$ $H_{y}(x, y, z-v t)=H_{y}(x, y, \zeta)$ :

$$
\frac{\partial^{2} H_{y}}{\partial \zeta^{2}}\left(1-\varepsilon \mu \beta^{2}\right)+\frac{\partial^{2} H_{y}}{\partial x^{2}}+\frac{\partial}{\partial y}\left(\frac{1}{\mu} \frac{\partial}{\partial y}\left[\mu H_{y}\right]\right)=-e v \frac{\partial n}{\partial x} .
$$

We introduce the operator

$$
\hat{T}_{H} H_{y}=\frac{1}{\left(1-\varepsilon \mu \beta^{2}\right)}\left[\frac{\partial^{2} H_{y}}{\partial x^{2}}+\frac{\partial}{\partial y}\left(\frac{1}{\mu} \frac{\partial}{\partial y}\left[\mu H_{y}\right]\right)\right] .
$$

We then can rewrite (19) as

$$
\frac{\partial^{2} H_{y}}{\partial \zeta^{2}}+\hat{T}_{H} H_{y}=\frac{-e v}{1-\varepsilon \mu \beta^{2}}\left(\frac{\partial n}{\partial x}\right) .
$$

We should stress here that Eqs. (15) and (22) are independent. Each equation corresponds to a specific type of propagating wave, (15) to longitudinal section magnetic $(L S M)$ modes, (22) to longitudinal section electric ( $L S E)$ modes.

\section{A. Transverse operator structure and transverse eigenfunctions}

If we assume that the charge distribution is a finite function inside the vacuum gap and

$$
\begin{gathered}
\varepsilon(y)= \begin{cases}1, & y \in[-b, b] \\
\varepsilon_{1}, & y \in[-c,-b) \cup(b, c],\end{cases} \\
\mu(y)= \begin{cases}1, & y \in[-b, b] \\
\mu_{1}, & y \in[-c,-b) \cup(b, c],\end{cases}
\end{gathered}
$$

then the boundary conditions for the $y$ components of the magnetic and electric fields on the conducting boundaries are

$$
\begin{gathered}
\left.E_{y}\right|_{x=0}=0 ;\left.\quad E_{y}\right|_{x=w}=0 ;\left.\quad \frac{\partial E_{y}}{\partial y}\right|_{y= \pm c}=0 ; \\
\left.\frac{\partial H_{y}}{\partial x}\right|_{x=0}=0 ;\left.\quad \frac{\partial H_{y}}{\partial x}\right|_{x=w}=0 ;\left.\quad H_{y}\right|_{y= \pm c}=0 ;
\end{gathered}
$$

and on the dielectric boundaries

$$
\begin{aligned}
& \left.E_{y}\right|_{y= \pm b \mp 0}=\left.\varepsilon_{1} E_{y}\right|_{y= \pm b \pm 0} ; \\
& \left.\frac{\partial E_{y}}{\partial y}\right|_{y= \pm b \mp 0}=\left.\frac{\partial E_{y}}{\partial y}\right|_{y= \pm b \pm 0} ; \\
& \left.\frac{\partial H_{y}}{\partial y}\right|_{y= \pm b \mp 0}=\left.\frac{\partial H_{y}}{\partial y}\right|_{y= \pm b \pm 0} ;
\end{aligned}
$$

We consider the eigenvalue equations for the operators $\hat{T}_{E}$ and $\hat{T}_{H}[(26)$ and (27)]:

$$
\begin{gathered}
\hat{T}_{E} \Psi_{E}(x, y)=\lambda_{E} \Psi_{E}(x, y), \quad \Psi_{E}(0, y)=0 ; \\
\Psi_{E}(w, y)=0 ;\left.\quad \frac{\partial \Psi_{E}(x, y)}{\partial y}\right|_{y=c}=0 ; \\
\left.\frac{\partial \Psi_{E}(x, y)}{\partial y}\right|_{y=-c}=0 \\
\hat{T}_{H} \Psi_{H}(x, y)=\lambda_{H} \Psi_{H}(x, y),\left.\quad \frac{\partial \Psi_{H}(x, y)}{\partial x}\right|_{x=0}=0 ; \\
\left.\frac{\partial \Psi_{H}(x, y)}{\partial x}\right|_{x=w}=0 ; \quad \Psi_{H}(x,-c)=0 ; \\
\Psi_{H}(x, c)=0 .
\end{gathered}
$$

Then we can introduce a series expansion ansatz for the transverse eigenfunctions $\Psi_{E}(x, y)$ and $\Psi_{H}(x, y)$ :

$$
\begin{aligned}
& \Psi_{E}(x, y)=\sum_{n=1}^{\infty} X_{E}^{n}(x) Y_{E}^{n}(y), \\
& \Psi_{H}(x, y)=\sum_{n=1}^{\infty} X_{H}^{n}(x) Y_{H}^{n}(y) .
\end{aligned}
$$

The eigenvalue problem for the $X$ functions is

$$
\begin{aligned}
\frac{\partial^{2}}{\partial x^{2}} X_{E, H}(x) & =-k_{x}^{2} X_{E, H}(x), \quad X_{E}(0)=0 ; \\
X_{E}(w) & =0 ; \quad \frac{\partial X_{H}(0)}{\partial x}=0 ; \quad \frac{\partial X_{H}(w)}{\partial x}=0 .
\end{aligned}
$$

Because of the self-adjointness of the operator defined by (30), the sets

$$
\left\{X_{E}(x)\right\}_{n}=\left\{\sin \left(k_{x}^{n} x\right)\right\}_{n} ; \quad\left\{X_{H}(x)\right\}_{n}=\left\{\cos \left(k_{x}^{n} x\right)\right\}_{n}
$$

define complete orthogonal systems with $k_{x}^{n}=\pi n / w$. This is of course the familiar Fourier series.

Substituting (28) into (26) and taking into account (25) we can write the equations for the transverse electric eigenfunctions as (suppressing the $n$ index for simplicity) 


$$
\begin{gathered}
\frac{1}{\left(1-\varepsilon \mu \beta^{2}\right)} \frac{\partial}{\partial y}\left(\frac{1}{\varepsilon} \frac{\partial \varepsilon Y_{E}(y)}{\partial y}\right)-\frac{k_{x}^{2}}{\left(1-\varepsilon \mu \beta^{2}\right)} Y_{E}(y)=\lambda_{E} Y_{E}(y), \quad \frac{\partial Y_{E}(-c)}{\partial y}=0 ; \quad \frac{\partial Y_{E}(c)}{\partial y}=0 ; \\
Y_{E}( \pm b \mp 0)=\varepsilon_{1} Y_{E}( \pm b \pm 0) ;\left.\quad \frac{\partial Y_{E}}{\partial y}\right|_{y= \pm b \mp 0}=\left.\frac{\partial Y_{E}}{\partial y}\right|_{y= \pm b \pm 0} \cdot
\end{gathered}
$$

Similarly, substituting (29) into (27) and taking into account (25) we get a set of analogous expressions for the transverse magnetic eigenfunctions:

$$
\begin{aligned}
\frac{1}{\left(1-\varepsilon \mu \beta^{2}\right)} \frac{\partial}{\partial y}\left(\frac{1}{\mu} \frac{\partial \mu Y_{H}(y)}{\partial y}\right)-\frac{k_{x}^{2}}{\left(1-\varepsilon \mu \beta^{2}\right)} Y_{H}(y)=\lambda_{H} Y_{H}(y), \quad Y_{H}(-c)=0 ; \quad Y_{H}(c)=0 . \\
Y_{H}( \pm b \mp 0)=\mu_{1} Y_{H}( \pm b \pm 0) ;\left.\quad \frac{\partial Y_{H}}{\partial y}\right|_{y= \pm b \mp 0}=\left.\frac{\partial Y_{H}}{\partial y}\right|_{y= \pm b \pm 0} .
\end{aligned}
$$

Because the material layers are assumed to be placed symmetrically relative to the OZX plane, the electric and magnetic eigenfunctions of (32) and (33) can be separated further into symmetric and asymmetric functions.

We can the write the solution to (32) as follows.

Symmetric eigenfunctions.-

$$
Y_{E, \mathrm{~s}}\left(y, \lambda_{E}\right)=A_{\mathrm{s}} \times \begin{cases}\frac{\cosh \left(k_{\mathrm{c}}^{E} b\right)}{\varepsilon_{1} \cos \left[k_{m d}^{E}(c-b)\right]} \cos \left[k_{m d}^{E}(c-y)\right], & b \leq y \leq c \\ \cosh \left(k_{\mathrm{ch}}^{E} y\right), & -b \leq y \leq b \\ \frac{\cosh \left(k_{\mathrm{c}}^{E} b\right)}{\varepsilon_{1} \cos \left[k_{m d}^{E}(c-b)\right]} \cos \left[k_{m d}^{E}(c+y)\right], & -c \leq y \leq-b .\end{cases}
$$

Asymmetric eigenfunctions.-

$$
Y_{E, \text { as }}\left(y, \lambda_{E}\right)=A_{\text {as }} \times \begin{cases}\frac{\sinh \left(k_{c h}^{E} b\right)}{\varepsilon_{1} \cos \left[k_{m d}^{E}(c-b)\right]} \cos \left[k_{m d}^{E}(c-y)\right], & b \leq y \leq c \\ \sinh \left(k_{\mathrm{ch}}^{E} y\right), & -b \leq y \leq b \\ -\frac{\sinh \left(k_{k \mathrm{~h}}^{E} b\right)}{\varepsilon_{1} \cos \left[k_{m d}^{E}(c-b)\right]} \cos \left[k_{m d}^{E}(c+y)\right], & -c \leq y \leq-b .\end{cases}
$$

Here we have defined the wave numbers in the vacuum channel and dielectric respectively as

$$
k_{\mathrm{ch}}^{E}=\sqrt{\left(1-\beta^{2}\right) \lambda_{E}+k_{x}^{2}}, \quad k_{m d}^{E}=\sqrt{\left(\varepsilon_{1} \mu_{1} \beta^{2}-1\right) \lambda_{E}-k_{x}^{2}} .
$$

The dispersion relations for the symmetric and asymmetric functions can then be written as

$$
\varepsilon_{1} k_{\mathrm{ch}}^{E} \tanh \left(k_{\mathrm{ch}}^{E} b\right)-k_{m d}^{E} \tan \left[k_{m d}^{E}(c-b)\right]=0, \quad \varepsilon_{1} k_{\mathrm{ch}}^{E} \operatorname{coth}\left(k_{\mathrm{ch}}^{E} b\right)-k_{m d}^{E} \tan \left[k_{m d}^{E}(c-b)\right]=0 .
$$

Eigenfunctions of the magnetic problem (33) also separate into symmetric and asymmetric parts.

Symmetric eigenfunctions are given by

$$
Y_{H, \mathrm{~s}}\left(y, \lambda_{H}\right)=B_{\mathrm{s}} \times \begin{cases}\frac{\cosh \left(k_{\mathrm{c}}^{H} b\right)}{\mu_{1} \sin \left[k_{m d}^{H}(c-b)\right]} \sin \left[k_{m d}^{H}(c-y)\right], & b \leq y \leq c \\ \cosh \left(k_{\mathrm{ch}}^{H} y\right), & -b \leq y \leq b \\ \frac{\cosh \left(k_{\mathrm{ch}}^{H} b\right)}{\mu_{1} \sin \left[k_{m d}^{H}(c-b)\right]} \sin \left[k_{m d}^{H}(c+y)\right], & -c \leq y \leq-b .\end{cases}
$$

Asymmetric eigenfunctions are given by

$$
Y_{H, \text { as }}\left(y, \lambda_{H}\right)=B_{\text {as }} \times \begin{cases}\frac{\sinh \left(k_{\mathrm{ch}}^{H} b\right)}{\mu_{1} \sin \left[k_{m d}^{H}(c-b)\right]} \sin \left[k_{m d}^{H}(c-y)\right], & b \leq y \leq c \\ \sinh \left(k_{\mathrm{ch}}^{H} y\right), & -b \leq y \leq b \\ -\frac{\sinh \left(k_{\mathrm{ch}}^{H} b\right)}{\mu_{1} \sin \left[k_{m d}^{H}(c-b)\right]} \sin \left[k_{m d}^{H}(c+y)\right], & -c \leq y \leq-b .\end{cases}
$$

Here we define

$$
k_{\mathrm{ch}}^{H}=\sqrt{\left(1-\beta^{2}\right) \lambda_{H}+k_{x}^{2}}, \quad k_{m d}^{H}=\sqrt{\left(\varepsilon_{1} \mu_{1} \beta^{2}-1\right) \lambda_{H}-k_{x}^{2}} .
$$

The dispersion relations for the symmetric and asymmetric functions respectively are given by 


$$
\begin{aligned}
& \mu_{1} k_{\mathrm{ch}}^{H} \tanh \left(k_{\mathrm{ch}}^{H} b\right)+k_{m d}^{H} \cot \left[k_{m d}^{H}(c-b)\right]=0, \\
& k_{m d}^{H} \tanh \left(k_{\mathrm{ch}}^{H} b\right)+\mu_{1} k_{\mathrm{ch}}^{H} \tan \left[k_{m d}^{H}(c-b)\right]=0 .
\end{aligned}
$$

\section{B. Expansion method}

Let us next examine the operator $\hat{T}_{E}(14)$. We consider the Hilbert space $\mathcal{H}_{E}=L_{2}\left\{[-c, c],\left|\varepsilon\left(1-\varepsilon \mu \beta^{2}\right)\right|\right\} \times$ $L_{2}([0, w])$, where $\rho=\left|\varepsilon\left(1-\varepsilon \mu \beta^{2}\right)\right|$ is the weight function. The scalar product in this space is given by

$$
(\psi, \varphi)_{E}=\int_{0}^{w} \int_{-c}^{c}\left|\varepsilon\left(1-\varepsilon \mu \beta^{2}\right)\right| \psi^{*} \varphi d x d y,
$$

where $\varphi, \psi \in \mathcal{H}_{E}$.

The metallic (24) and dielectric boundary conditions (25) lead to

$$
\begin{aligned}
\operatorname{Dom}\left(\hat{T}_{E}\right)= & \left\{\varphi \in \mathcal{H}_{E}: \partial_{y} \varphi(x, \pm c)=0 ;\right. \\
& \varphi(0, y)=\varphi(w, y)=0 ; \\
& \varphi(x, \pm b \mp 0)=\varepsilon_{1} \varphi(x, \pm b \pm 0) ; \\
& \left.\partial_{y} \varphi(x, \pm b \mp 0)=\partial_{y} \varphi(x, \pm b \pm 0)\right\} .
\end{aligned}
$$

[Dom $(X)$ indicates the domain of the operator $X$.]

The adjoint operator $\hat{T}_{E}{ }^{*}$ can be found using the definition

$$
\left(\psi, \hat{T}_{E} \varphi\right)_{E}-\left(\hat{T}_{E}^{*} \psi, \varphi\right)_{E}=0 .
$$

This leads to

$$
\begin{aligned}
\operatorname{Dom}\left(\hat{T}_{E}^{*}\right)= & \left\{\varphi \in \mathcal{H}_{E}: \partial_{y} \varphi(x, \pm c)=0\right. \\
& \varphi(0, y)=\varphi(w, y)=0 \\
& \varphi(x, \pm b \mp 0)=-\varepsilon_{1} \varphi(x, \pm b \pm 0) \\
& \left.\partial_{y} \varphi(x, \pm b \mp 0)=-\partial_{y} \varphi(x, \pm b \pm 0)\right\} .
\end{aligned}
$$

Following the same steps for the operator $\hat{T}_{H}$ (21), we introduce the Hilbert space $\mathcal{H}_{H}=L_{2}\{[-c, c]$, $\left.\left|\mu\left(1-\varepsilon \mu \beta^{2}\right)\right|\right\} \times L_{2}([0, w])$, with the scalar product

$$
(\psi, \varphi)_{H}=\int_{0}^{w} \int_{-c}^{c}\left|\mu\left(1-\varepsilon \mu \beta^{2}\right)\right| \psi^{*} \varphi d x d y,
$$

where $\varphi, \psi \in \mathcal{H}_{H}$.

Applying the boundary conditions (24) and (25) we get

$$
\begin{aligned}
\operatorname{Dom}\left(\hat{T}_{H}\right)= & \left\{\varphi \in \mathcal{H}_{H}: \varphi(x, \pm c)=0 ;\right. \\
& \varphi(0, y)=\varphi(w, y)=0 ; \\
& \varphi(x, \pm b \mp 0)=\mu_{1} \varphi(x, \pm b \pm 0) ; \\
& \left.\partial_{y} \varphi(x, \pm b \mp 0)=\partial_{y} \varphi(x, \pm b \pm 0)\right\} .
\end{aligned}
$$

Using the definition of the adjoint (42), the adjoint operator's domain is found to be

$$
\begin{aligned}
\operatorname{Dom}\left(\hat{T}_{H}^{*}\right)= & \left\{\varphi \in \mathcal{H}_{H}: \varphi(x, \pm c)=0\right. \\
& \varphi(0, y)=\varphi(w, y)=0 \\
& \varphi(x, \pm b \mp 0)=-\mu_{1} \varphi(x, \pm b \pm 0) \\
& \left.\partial_{y} \varphi(x, \pm b \mp 0)=-\partial_{y} \varphi(x, \pm b \pm 0)\right\} .
\end{aligned}
$$

It immediately follows that $\operatorname{Dom}\left(\hat{T}_{E}\right) \neq \operatorname{Dom}\left(\hat{T}_{E}^{*}\right)$ and $\operatorname{Dom}\left(\hat{T}_{H}\right) \neq \operatorname{Dom}\left(\hat{T}_{H}^{*}\right)$, which means that the operators $\hat{T}_{E}$ and $\hat{T}_{H}$ are not self-adjoint in $\mathcal{H}_{E}$ and $\mathcal{H}_{H}$, respectively, so the eigenfunctions are not guaranteed to form a complete orthogonal set. In turn this would imply that all solutions for $\Psi_{E}\left(\Psi_{H}\right)$ are not necessarily given by (28), (34), and (35) [(29), (38), and (39)]. However, it can be proved that the eigenfunctions of the tasks (26) and (27) along with the conditions (25) form Riesz bases in $\mathcal{H}_{E}$ and $\mathcal{H}_{H}$, respectively. This means that for the sets $\left\{\Psi_{E}(x, y)\right\}$ and $\left\{\Psi_{H}(x, y)\right\}$ there exist bounded and boundedly invertible operators $\hat{X}_{E}$ and $\hat{X}_{H}$ such that $e_{E}=\hat{X}_{E}^{-1} \Psi_{E}(x, y)$ and $e_{H}=\hat{X}_{H}^{-1} \Psi_{H}(x, y)$, where $\left\{e_{E}\right\}$ and $\left\{e_{H}\right\}$ are orthogonal bases in $\mathcal{H}_{E}$ and $\mathcal{H}_{H}$, respectively. (The proof of this is rather involved; we provide an outline of the proof in the Appendix. A paper containing the complete proof will be submitted to a journal with a more mathematical focus.) Hence, any $f \in \mathcal{H}_{E}$ and any $g \in \mathcal{H}_{H}$ can be expanded as

$$
f=\sum\left(\Phi_{E}, f\right)_{E} \Psi_{E}, \quad g=\sum\left(\Phi_{H}, f\right)_{H} \Psi_{H} .
$$

Here $\left\{\Phi_{E}\right\}$ are eigenfunctions of $\hat{T}_{E}^{*}$ and $\left\{\Phi_{H}\right\}$ are eigenfunctions of $\hat{T}_{H}^{*}$. Because $\varepsilon$ and $\mu$ are bounded on $[-c, c]$ and $\varepsilon, \mu \in L_{1}([-c, c])$, metrics on $\mathcal{H}_{E}$ and $\mathcal{H}_{H}$ are equivalent to the metrics on $L_{2}([-c, c]) \times$ $L_{2}([0, w])$. Hence, the expansions (47) hold for $f, g \in$ $L_{2}([-c, c]) \times L_{2}([0, w])$. However, we should stress here that the conditions (25) hold only for finite functions on the intervals $[-c,-b],[-b, b],[b, c]$; in other words the excitation charge distribution should vanish at material boundaries.

The functions $\left\{\Psi_{E}\right\},\left\{\Phi_{E}\right\}$ and $\left\{\Psi_{H}\right\},\left\{\Phi_{H}\right\}$ satisfy the biorthogonality conditions

$$
\begin{aligned}
\left(\Phi_{E}^{n}, \Psi_{E}^{m}\right)_{E} & =\int\left|\varepsilon\left(1-\varepsilon \mu \beta^{2}\right)\right|\left(\Phi_{E}^{n}\right)^{*} \Psi_{E}^{m} d x d y=A_{E}^{n, m} \delta_{n, m}, \\
\left(\Phi_{H}^{n}, \Psi_{H}^{m}\right)_{H} & =\int\left|\mu\left(1-\varepsilon \mu \beta^{2}\right)\right|\left(\Phi_{H}^{n}\right)^{*} \Psi_{H}^{m} d x d y=A_{H}^{n, m} \delta_{n, m} .
\end{aligned}
$$

Because of the fact that

$$
\Phi_{E}=\hat{J} \Psi_{E}, \quad \Phi_{H}=\hat{J} \Psi_{H},
$$

where

$$
\hat{J}= \begin{cases}1, & y \in[-b, b] \\ -1, & y \in[-c,-b) \cup(b, c],\end{cases}
$$

one can write 


$$
\begin{aligned}
\left(\Psi_{E}^{n}, \hat{J} \Psi_{E}^{m}\right)_{E} & =\int \varepsilon\left(1-\varepsilon \mu \beta^{2}\right)\left(\Psi_{E}^{n}\right)^{*} \Psi_{E}^{m} d x d y=A_{E}^{n, m} \delta_{n, m}, \\
\left(\Psi_{H}^{n}, \hat{J} \Psi_{H}^{m}\right) & =\int \mu\left(1-\varepsilon \mu \beta^{2}\right)\left(\Psi_{H}^{n}\right)^{*} \Psi_{H}^{m} d x d y=A_{H}^{n, m} \delta_{n, m},
\end{aligned}
$$

which corresponds to a result from [29] of the so-called mode orthogonality condition

$$
\begin{aligned}
& \int \varepsilon\left(\varepsilon \mu \beta^{2}-1\right) E_{y m, n} E_{y p, q}^{*} d x d y=\Pi_{m, n}^{\mathrm{LSM}} \delta_{m p} \delta_{n q}, \\
& \int \mu\left(\varepsilon \mu \beta^{2}-1\right) H_{y m, n} H_{y p, q}^{*} d x d y=\Pi_{m, n}^{\mathrm{LSE}} \delta_{m p} \delta_{n q} .
\end{aligned}
$$

\section{SOLUTIONS FOR $E_{y}$ AND $H_{y}$}

Assuming a point charge passing through the structure at transverse coordinates $\left(x_{0}, y_{0}\right)$, Fourier transformation of (15) with respect to $\zeta$ leads to

$$
\begin{aligned}
& \hat{T}_{E}\left[\tilde{E}_{y}(x, y, k)\right]-k^{2} \tilde{E}_{y}(x, y, k) \\
& \quad=\frac{2 q \delta\left(x-x_{0}\right)}{\left(1-\varepsilon \mu \beta^{2}\right)} \frac{\partial}{\partial y}\left[\frac{\delta\left(y-y_{0}\right)}{\varepsilon}\right] .
\end{aligned}
$$

We introduce the normalized eigenfunctions

$$
\begin{aligned}
\psi_{E}^{\lambda_{n}}(x, y) & =\frac{1}{\sqrt{A_{n, n}}} \Psi_{E}^{\lambda_{n}}(x, y), \\
\phi_{E}^{\lambda_{n}}(x, y) & =\frac{1}{\sqrt{A_{n, n}}} \Phi_{E}^{\lambda_{n}}(x, y), \\
{\left[\phi_{E}^{\lambda_{n}}(x, y), \psi_{E}^{\lambda_{n}}(x, y)\right]_{E} } & =\delta_{n, m} .
\end{aligned}
$$

We then expand the transverse electric field (assuming $y_{0} \neq \pm b$ )

$$
\begin{aligned}
\tilde{E}_{y}^{\lambda}(k) & =\left(\phi_{E}^{\lambda}(x, y), \tilde{E}_{y}(x, y, k)\right)_{E}, \\
\tilde{E}_{y}(x, y, k) & =\sum \tilde{E}_{y}^{\lambda}(k) \psi_{E}^{\lambda}(x, y), \\
\frac{\delta\left(x-x_{0}\right)}{\left(1-\varepsilon \mu \beta^{2}\right)} \frac{\partial}{\partial y}\left[\frac{\delta\left(y-y_{0}\right)}{\varepsilon}\right] & =-\sum \frac{\partial\left[\phi_{E}^{\lambda}\left(x_{0}, y_{0}\right)\right]^{*}}{\partial y} \psi_{E}^{\lambda}(x, y) .
\end{aligned}
$$

Substituting (55) into (53), one can write for the region $-b<y_{0}<b$,

$$
\tilde{E}_{y}^{\lambda}(k)=-2 q \frac{\partial\left[\phi_{E}^{\lambda}\left(x_{0}, y_{0}\right)\right]^{*}}{\partial y} \frac{1}{\lambda_{E}-k^{2}} .
$$

Performing the inverse Fourier transform for $\zeta<0$ (field behind the bunch) leads to a solution in the form

$$
\begin{aligned}
E_{y}(x, y, \zeta)= & 2 \pi q \sum_{\lambda_{E}} \frac{\partial\left[\phi_{E}^{\lambda_{E}}\left(x_{0}, y_{0}\right)\right]^{*}}{\partial y} \psi_{E}^{\lambda_{E}}(x, y) \\
& \times\left[\frac{\exp \left(-\sqrt{\left|\lambda_{E}^{-}\right|} \zeta \mid\right)}{\sqrt{\left|\lambda_{E}^{-}\right|}}-2 \frac{\sin \left(\sqrt{\lambda_{E}^{+}}|\zeta|\right)}{\sqrt{\lambda_{E}^{+}}}\right],
\end{aligned}
$$

where $\lambda_{E}^{+}$refers to the positive eigenvalues $\left(\lambda_{E}>0\right)$ of $\hat{T}_{E}$ and $\lambda_{E}^{-}$to the negative eigenvalues $\left(\lambda_{E}<0\right)$ of $\hat{T}_{E}$.

By a similar process applied to (22) we obtain the solution for $H_{y}$. For the case $\zeta<0,-b<y_{0}<b$ we can write

$$
\begin{aligned}
H_{y}(x, y, \xi)= & 2 \pi q \beta \sum_{\lambda_{H}} \frac{\partial\left[\phi_{H}^{\lambda_{H}}\left(x_{0}, y_{0}\right)\right]^{*}}{\partial x} \psi_{H}^{\lambda_{H}}(x, y) \\
& \times\left[\frac{\exp \left(-\sqrt{\left|\lambda_{H}^{-}\right|}|\zeta|\right)}{\sqrt{\left|\lambda_{H}^{-}\right|}}-2 \frac{\sin \left(\sqrt{\lambda_{H}^{+}}|\zeta|\right)}{\sqrt{\lambda_{H}^{+}}}\right],
\end{aligned}
$$

where $\lambda_{H}^{+}$refers to positive eigenvalues $\left(\lambda_{H}>0\right)$ of $\hat{T}_{H}$ and $\lambda_{H}^{-}$to negative eigenvalues $\left(\lambda_{H}<0\right)$ of $\hat{T}_{H} ; \phi_{H}^{\lambda_{H}}$ and $\psi_{H}^{\lambda_{H}}$ are normalized eigenfunctions of $\hat{T}_{H}^{*}$ and $\hat{T}_{H}$, respectively.

\section{ACCELERATING FIELD AND DEFLECTING FORCE}

By Fourier transformation with respect to $\zeta$ of the Maxwell equations (1) and (2), we can express the transverse fields in terms of the longitudinal fields as

$$
\begin{aligned}
& i k\left[1-\varepsilon \mu \beta^{2}\right] \tilde{E}_{x}=\left[\frac{\partial \tilde{E}_{z}}{\partial x}+\mu \beta \frac{\partial \tilde{H}_{z}}{\partial y}\right], \\
& i k\left[1-\varepsilon \mu \beta^{2}\right] \tilde{E}_{y}=\left[\frac{\partial \tilde{E}_{z}}{\partial y}-\mu \beta \frac{\partial \tilde{H}_{z}}{\partial x}\right], \\
& i k\left[1-\varepsilon \mu \beta^{2}\right] \tilde{H}_{x}=\left[\frac{\partial \tilde{H}_{z}}{\partial x}-\varepsilon \beta \frac{\partial \tilde{E}_{z}}{\partial y}\right], \\
& i k\left[1-\varepsilon \mu \beta^{2}\right] \tilde{H}_{y}=\left[\frac{\partial \tilde{H}_{z}}{\partial y}+\varepsilon \beta \frac{\partial \tilde{E}_{z}}{\partial x}\right] .
\end{aligned}
$$

Combining the equations for $\tilde{E}_{y}$ and $\tilde{H}_{y}$ we obtain

$$
\begin{aligned}
& \frac{\partial^{2} \tilde{E}_{z}}{\partial y^{2}}+\varepsilon \mu \beta^{2} \frac{\partial^{2} \tilde{E}_{z}}{\partial x^{2}}-\beta \frac{\partial \tilde{H}_{z}}{\partial x} \frac{\partial \mu}{\partial y} \\
& \quad=i k\left[\frac{\partial}{\partial y}\left(\left[1-\varepsilon \mu \beta^{2}\right] \tilde{E}_{y}\right)+\mu \beta\left[1-\varepsilon \mu \beta^{2}\right] \frac{\partial \tilde{H}_{y}}{\partial x}\right] .
\end{aligned}
$$

Now let us consider the Helmholtz equation for the $\tilde{E}_{z}$ components 


$$
\begin{aligned}
\Delta_{x, y} \tilde{E}_{z}+i k \frac{\tilde{E}_{y}}{\varepsilon} \frac{\partial \varepsilon}{\partial y}-i k \beta \tilde{H}_{x} \frac{\partial \mu}{\partial y}-k^{2}\left[1-\varepsilon \mu \beta^{2}\right] \tilde{E}_{z} \\
=\frac{2 q i k}{\varepsilon}\left[1-\varepsilon \mu \beta^{2}\right] \tilde{n} .
\end{aligned}
$$

Substitution of $\frac{\partial^{2} \tilde{E}_{y}}{\partial y^{2}}$ from (63) and $\tilde{H}_{x}$ from (61) into (64) with $\tilde{E}_{y}$ from (60) gives

$$
\frac{\partial^{2} \tilde{E}_{z}}{\partial x^{2}}-k^{2} \tilde{E}_{z}=-i k\left(\frac{\partial \tilde{E}_{y}}{\partial y}+\frac{\tilde{E}_{y}}{\varepsilon} \frac{\partial \varepsilon}{\partial y}\right)+i k \frac{2}{\varepsilon} \tilde{n}-i k \mu \beta \frac{\partial \tilde{H}_{y}}{\partial x} .
$$

Now we consider the $\tilde{E}_{y}$ Helmholtz equation:

$$
\left[\frac{\partial^{2}}{\partial x^{2}}+\frac{\partial}{\partial y}\left[\frac{1}{\varepsilon} \frac{\partial}{\partial y}[\varepsilon \cdot]\right]\right] \tilde{E}_{y}-\left[1-\varepsilon \mu \beta^{2}\right] k^{2} \tilde{E}_{y}=2 \frac{\partial}{\partial y}\left[\frac{\tilde{n}}{\varepsilon}\right] .
$$

Integrating this equation with respect to $y$, we have

$$
\int\left[\frac{\partial^{2}}{\partial x^{2}}-\left[1-\varepsilon \mu \beta^{2}\right] k^{2}\right] \tilde{E}_{y} d y+\frac{1}{\varepsilon} \frac{\partial}{\partial y}\left[\varepsilon \tilde{E}_{y}\right]+C=2 \frac{\tilde{n}}{\varepsilon}
$$

Multiplication of this equation by $i k$ and substitution into (65) leads to

$$
\begin{aligned}
\frac{\partial^{2} \tilde{E}_{z}}{\partial x^{2}}-k^{2} \tilde{E}_{z}= & i k \int\left[\frac{\partial^{2}}{\partial x^{2}}-\left[1-\varepsilon \mu \beta^{2}\right] k^{2}\right] \tilde{E}_{y} d y \\
& -i k \mu \beta \frac{\partial \tilde{H}_{y}}{\partial x} .
\end{aligned}
$$

Using the boundary condition on the conductors $\tilde{E}_{z}( \pm c)=0$, we find the constant of integration $C=0$.

For the boundary conditions on the sides of the structure, $E_{z}(x=0)=0, E_{z}(x=a)=0$, we can expand $\tilde{E}_{z}$ in the series

$$
\tilde{E}_{z}=\sum X_{n}^{E}(x) E_{z}^{n}(y)
$$

Taking into account that

$\tilde{E}_{y}=-2 q X_{n}^{E}(x) X_{n}^{E}\left(x_{0}\right) \frac{E_{y}^{n}}{\lambda_{E}-k^{2}}, \quad E_{y}^{n}=\frac{\partial Y_{E}^{*}\left(y_{0}\right)}{\partial y} Y_{E}(y)$,

where $Y_{E}^{*}$ is the conjugate eigenfunction of the adjoint problem (32), and

$$
\begin{aligned}
\tilde{H}_{y} & =-2 q \beta \lambda_{x}^{n} X_{n}^{H}(x) X_{n}^{E}\left(x_{0}\right) \frac{k_{x} H_{y}^{n}}{\lambda_{H}-k^{2}}, \\
H_{y}^{n} & =Y_{H}^{*}\left(y_{0}\right) Y_{H}(y),
\end{aligned}
$$

where $Y_{H}^{*}$ is the conjugate eigenfunction of the adjoint problem (33). Substitution of (69)-(71) into (68) gives

$$
\begin{aligned}
E_{z}^{n}= & -\frac{2 q X_{E}^{n}\left(x_{0}\right)}{\left(k_{x}^{n}\right)^{2}+k^{2}} \frac{i k}{\lambda_{E}-k^{2}} \frac{\left[1-\varepsilon \mu \beta^{2}\right] k^{2}+\left(k_{x}^{n}\right)^{2}}{k_{y}(y)} \\
& \times I\left[E_{y}^{n}\right]\left[k_{y}(y) y\right]+\frac{2 q \mu \beta^{2} X_{E}^{n}\left(x_{0}\right)}{\left(k_{x}^{n}\right)^{2}+k^{2}} \frac{i k\left(k_{x}^{n}\right)^{2}}{\lambda_{H}-k^{2}} H_{y}^{n} .
\end{aligned}
$$

Here we define

$$
k_{y}(y)=\sqrt{\left[1-\varepsilon \mu \beta^{2}\right] \lambda_{E}+\left(k_{x}^{n}\right)^{2}}
$$

and denote for brevity the integral by the operation $I$ :

$$
I[f](y) \equiv \int f(y) d y, \quad \frac{d}{d y} I[f](y)=f(y) .
$$

Using the inverse Fourier transformation the Cherenkov radiation field (only real poles contributing for $\xi<0$ and only positive eigenvalues of $\hat{T}_{E}$ and $\hat{T}_{H}$ ) can be found as

$$
\begin{aligned}
E_{z}(x, y, \xi)= & 4 \pi q \sum_{n} X_{E}^{n}\left(x_{0}\right) X_{E}^{n}(x) \\
& \times\left[\sum_{\lambda_{E}} \frac{k_{y} I\left[E_{y}^{n}\right]\left[k_{y}(y) y\right]}{\lambda_{E}+\left(k_{x}^{n}\right)^{2}} \cos \left(\sqrt{\lambda_{E}}|\xi|\right)\right. \\
& \left.-\mu \beta^{2} \sum_{\lambda_{H}} \frac{\left(k_{x}^{n}\right)^{2} H_{y}^{n}}{\lambda_{H}+\left(k_{x}^{n}\right)^{2}} \cos \left(\sqrt{\lambda_{H}}|\xi|\right)\right] .
\end{aligned}
$$

The transverse force inside the channel can be found using the Panofsky-Wenzel theorem:

$$
\frac{\partial \mathbf{F}_{\perp}}{\partial z}=e \nabla_{\perp} E_{z}
$$

Hence,

$$
F_{x}=\int \frac{\partial E_{z}}{\partial x} d z, \quad F_{y}=\int \frac{\partial E_{z}}{\partial y} d z .
$$

Substitution of $E_{z}$ leads to the full expressions for the transverse force components:

$$
\begin{gathered}
F_{x}(x, y, \xi)=4 \pi q \sum_{n} X_{E}^{n}\left(x_{0}\right) \frac{\partial X_{E}^{n}(x)}{\partial x}\left[\sum_{\lambda_{E}} \frac{k_{y} I\left[E_{y}^{n}\right]\left[k_{y}(y) y\right]}{\lambda_{E}+\left(k_{x}^{n}\right)^{2}} \frac{\sin \left(\sqrt{\lambda_{E}}|\xi|\right)}{\sqrt{\lambda_{E}}}-\mu \beta^{2} \sum_{\lambda_{H}} \frac{\left(k_{x}^{n}\right)^{2} H_{y}^{n}}{\lambda_{H}+\left(k_{x}^{n}\right)^{2}} \frac{\sin \left(\sqrt{\lambda_{H}}|\xi|\right)}{\sqrt{\lambda_{H}}}\right], \\
F_{y}(x, y, \xi)=4 \pi q \sum_{n} X_{E}^{n}\left(x_{0}\right) X_{E}^{n}(x)\left[\sum_{\lambda_{E}} \frac{\left(k_{y}\right)^{2} E_{y}^{n}}{\lambda_{E}+\left(k_{x}^{n}\right)^{2}} \frac{\sin \left(\sqrt{\lambda_{E}}|\xi|\right)}{\sqrt{\lambda_{E}}}-\mu \beta^{2} \sum_{\lambda_{H}} \frac{\left(k_{x}^{n}\right)^{2}}{\lambda_{H}+\left(k_{x}^{n}\right)^{2}} \frac{\partial H_{y}^{n}}{\partial y} \frac{\sin \left(\sqrt{\lambda_{H}}|\xi|\right)}{\sqrt{\lambda_{H}}}\right] .
\end{gathered}
$$


TABLE I. DLA structure geometries and electron beam parameters used for the comparison of the theory developed in this paper and wakefield experiments carried out at the Argonne Wakefield Accelerator at ANL [38,39] and the Accelerator Test Facility at BNL $[14,40]$.

\begin{tabular}{lcccccccc}
\hline \hline Experiment/Ref. & $f, \mathrm{GHz}, \mathrm{LSM}_{11}$ & $\varepsilon$ & $w, \mathrm{~cm}$ & $b, \mathrm{~mm}$ & $c, \mathrm{~mm}$ & Energy, MeV & $\sigma_{z, \mathrm{~mm}}$ & $Q$ \\
\hline ANL/AWA [38,39] & 25 & 5.7 & 0.8 & 2 & 3.19 & 15 & $1.5-2.5$ & $70 \mathrm{nC}$ \\
BNL/ATF [40] & 170 & 3.8 & 2 & 0.12 & 0.36 & 59 & 0.12 & $90 \mathrm{pC}$ \\
BNL/ATF [14] & 295 & $5.7 / 1.8$ & 0.1 & 0.10 & 0.25 & 57 & 0.10 & $70 \mathrm{pC}$ \\
\hline \hline
\end{tabular}

\section{COMPARISON WITH NUMERICAL SIMULATIONS AND EXPERIMENTAL RESULTS}

The formalism presented above allows obtaining a rigorous full solution for the wakefields generated by charged particle bunches passing along a rectangular accelerating structure loaded with symmetric dielectric slabs. Note that this analysis allows the permittivity in one slab to be an arbitrary function of $y$ as long as the distribution in the other slab is mirrored across the central $x-z$ plane. Comprehensive wakefield analysis and optimization of the structure parameters require electric and magnetic field magnitudes and spectra to be evaluated and used in the expressions for the accelerating gradient. The forces deflecting the electron bunch and the amplitudes of the electric and magnetic fields on the inner surfaces of the structure are also of importance for any practical accelerator based on this technology. To solve this problem using the formalism described above, the code RECTANGULAR was developed, and the results of computations based on the code using the analytic approach developed here are both in good agreement with the results of analyses based on traditional numerical codes like $\mathrm{CST}^{\mathrm{TM}}$ [37].

Wakefields in rectangular dielectric-loaded structures have been experimentally studied [14,38-40], Table I. In [38,39] a wakefield measurement using a single crystal diamond-loaded rectangular accelerating structure was reported. Diamond was proposed as a material for dielectricloaded accelerating (DLA) structures a few years ago $[18,19]$. It has a very low microwave loss tangent, the highest available coefficient of thermal conductivity, and high rf breakdown field [17]. In [38,39], the high charge beam from the argon wakefield accelerator (AWA) linac $\left(\sim 70 \mathrm{nC}, \sigma_{z}=1.5-2.5 \mathrm{~mm}\right)$ was transported through a rectangular diamond-loaded resonator and induced an intense wakefield behind the bunch.

The expressions derived above were used for analyzing the wakefields generated by a Gaussian relativistic electron bunch with the parameters of the Argon Wakefield AcceleratorAWA in this structure- $w=8 \mathrm{~mm}, \quad b=$ $2 \mathrm{~mm}, c=3.19 \mathrm{~mm}$, and $\varepsilon_{1}=5.7$. The dependence of the $E_{z}$ component as a function of the distance $\zeta=z-v t$ behind it is shown in Fig. 2. The bunch is located at point $x_{0}=w / 2, y_{0}=0, \zeta_{0}=8 \mathrm{~cm}$; the observation point is $x=w / 2, y=0$. The results of computations based on the theory developed here (dotted curve computed using the RECTANGULAR code) show good agreement with the results of numerical calculations of fields in the same structure using the CST PARTICLE STUDIO ${ }^{\mathrm{TM}}$ code (solid) [37]. Small difference between curves in Fig. 2 is connected with limitation of mode quantity and taking into account here only the Cherenkov part of radiation.

In [40], wakefield acceleration of a relativistic electron beam in a dielectric-lined slab-symmetric $\mathrm{THz}$ structure was reported. The high energy tail of a $\sim 60 \mathrm{MeV}$ electron beam was accelerated by $\sim 150 \mathrm{keV}$ in a $2 \mathrm{~cm}$ long, slabsymmetric quartz $(\varepsilon=3.8)$ structure. This split-bunch distribution was verified by a longitudinal reconstruction analysis of the emitted coherent transition radiation. The dielectric waveguide structure was characterized by spectral analysis of the Cherenkov radiation at $\mathrm{THz}$ frequencies. The spacing of a relativistic bunch train was selectively tuned to excite the second longitudinal mode $L S M_{21}$ [40]. The structure parameters are $b=120 \mu \mathrm{m}$, $c=360 \mu \mathrm{m}$, structure width $w=2 \mathrm{~cm}$, structure length $L=2 \mathrm{~cm}$, bunch charge $Q=90 \mathrm{pC}$, bunch length $\sigma_{z}=120 \mu \mathrm{m}, \quad \sigma_{x}=5 \mathrm{~mm}, \quad \sigma_{y}=0$; bunch energy $W=59 \mathrm{MeV}$.

Figure 3(a) shows the $E_{z}$ field spectrum and Fig. 3(b) shows the $E_{z}$ magnitude behind the bunch with the structure parameters [40]. It should be noted that the only

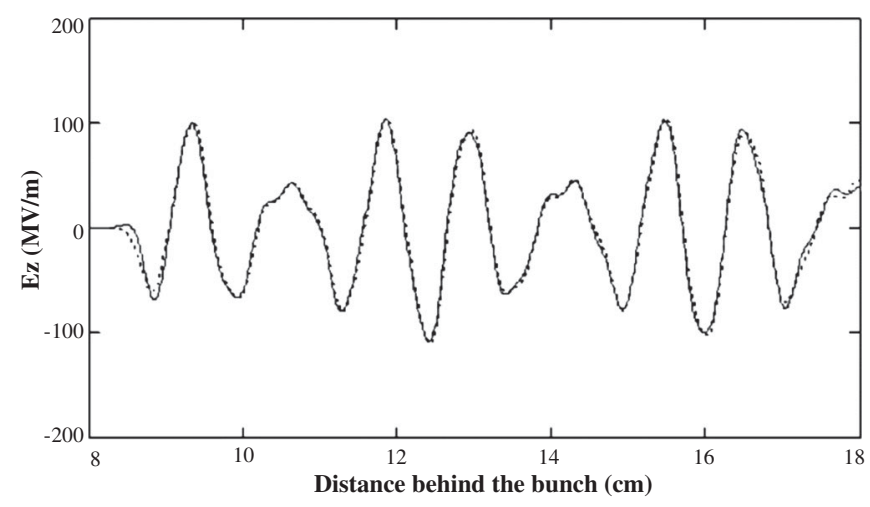

FIG. 2. Longitudinal electric field behind the bunch in a $25 \mathrm{GHz}$ diamond based rectangular accelerating structure with $w=8 \mathrm{~mm}, a=2 \mathrm{~mm}, b=3.19 \mathrm{~mm}$, and $\varepsilon_{1}=5.7$. The bunch has a Gaussian charge distribution, energy $W=15 \mathrm{MeV}$, charge $Q=100 \mathrm{nC}$, and length $\sigma_{z}=1.5 \mathrm{~mm}$. The solid curve is obtained using the PARTICLE STUDIO CST ${ }^{\mathrm{TM}}$ code [37]; the dotted curve is computed using the RECTANGULAR code [38,39]. 


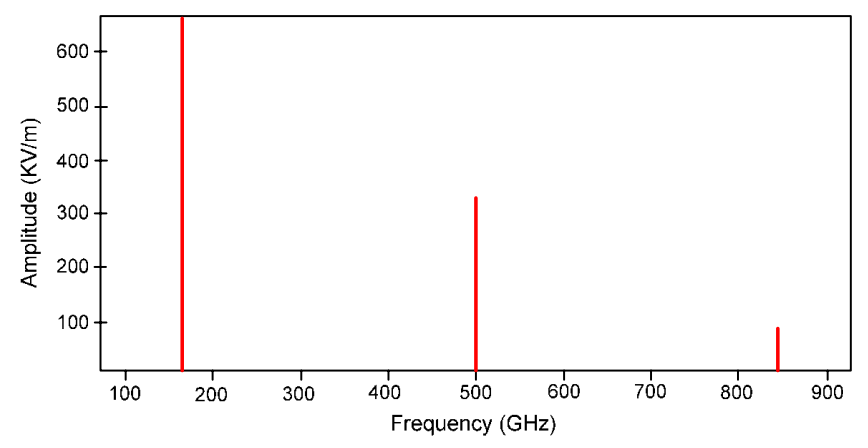

(a)

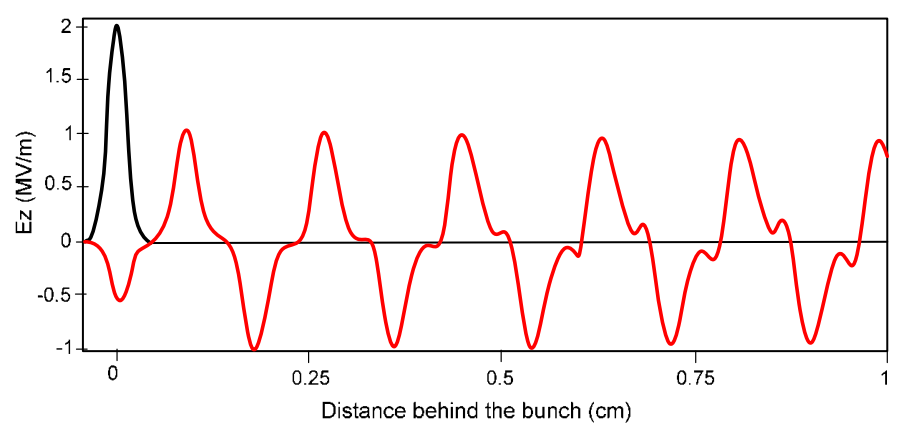

(b)

FIG. 3. (a) $E_{z}$ field spectrum and (b) lineout of the field behind the bunch (black curve) for the $L S M_{1,1}, L S M_{2,1}$, and $L S M_{3,1}$ asymmetric modes, $\sigma_{x}=5 \mathrm{~mm}, \sigma_{y}=0$. Frequencies are in good agreement with [40] $\lambda_{1}=1800 \mu \mathrm{m}, \lambda_{2}=600 \mu \mathrm{m}$, and $\lambda_{3}=$ $355 \mu \mathrm{m}$. (For this and subsequent figures, see Ref. [40] and Table I for further details.)

asymmetric $L S M_{1,1}, L S M_{2,1}$, and $L S M_{3,1}$ modes can be excited by the (relatively wide in $X$ ) bunch $\sigma_{x}=5 \mathrm{~mm}$, $\sigma_{y}=0$. These mode frequencies are in full agreement with [40] that gives $\lambda_{1}=1800 \mu \mathrm{m}, \lambda_{2}=600 \mu \mathrm{m}$, and $\lambda_{3}=$ $355 \mu \mathrm{m}$ for the mode wavelengths. Meanwhile, the proposed formalism allows resolving the fine structure of the $L S M$ modes: Fig. 4 shows the $E_{z}$ field spectrum for the wider $L S M_{i, j}$ mode spectrum, where $L S M_{1,1}, L S M_{2,1}$, and $L S M_{3,1}$ are shown for the narrower bunch width $\sigma_{x}=$ $500 \mu \mathrm{m}, \sigma_{y}=0$. The bar groups near the base $L S M_{1,1}$, $L S M_{2,1}$, and $L S M_{3,1}$ frequencies correspond to higher $k_{x}$ values of each mode and can be written as $L S M_{1,2}$, $L S M_{1,3} \ldots ; L S M_{2,2}, L S M_{2,3} \ldots ; L S M_{3,2}, L S M_{3,3}$ modes correspondingly. Figure 5 presents the $E_{z}$ field spectrum of the symmetric $L S E_{i, j}$ modes for the same bunch width $\sigma_{x}=500 \mu \mathrm{m}, \sigma_{y}=0$, where the bar groups now correspond to higher $k_{x}$ values. Figure 6(a) shows the spectrum of the $E_{z}$ field and Fig. 6(b) presents the $E_{z}$ field for the bunch width $\sigma_{x}=500 \mu \mathrm{m}, \sigma_{y}=0$. The vertical deflecting force $F_{y}$ spectrum and magnitude for a $40 \mu \mathrm{m}$ offset are shown in Fig. 7. The only $L S M_{1,1}$ and $L S M_{1,2}$

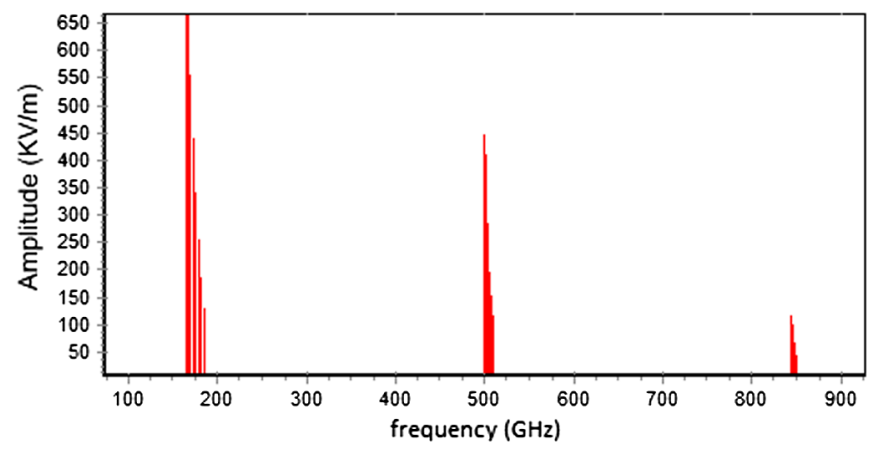

FIG. 4. $E_{z}$ field spectrum for the $L S M_{i, j}$ modes. $L S M_{1,1}$, $L S M_{2,1}$, and $L_{S M}$ modes are shown for $\sigma_{x}=500 \mu \mathrm{m}, \sigma_{y}=0$. Bar groups near LSM mode frequencies correspond to higher $k_{x}$ values for each mode: $L S M_{1,2}, L S M_{1,3}$; $\operatorname{LSM}_{2,2}, \mathrm{LSM}_{2,3} ; \mathrm{LSM}_{3,2}, \mathrm{LSM}_{3,3}$, respectively. modes are excited for $\sigma_{x}=5 \mathrm{~mm}, \sigma_{y}=0$ bunch width. It is worth mentioning than the mode spectrum consists of an extremely low frequency component at $7.6 \mathrm{GHz}$ in comparison with the $L S M_{1,1}$ mode frequency of $167 \mathrm{GHz}$ but this low frequency mode is only weakly excited. The fine structure of the $F_{y}$ field spectrum in the range of $10-700 \mathrm{GHz}$ is presented in Fig. 8(a) for $40 \mu \mathrm{m}$ offset; $L S M_{1, j}$ symmetric modes dominate for $\sigma_{x}=500 \mu \mathrm{m}$, $\sigma_{y}=0$. The transverse deflecting field magnitude behind the bunch for a $40 \mu \mathrm{m}$ offset is shown in Fig. 8(b) for the $L S M_{1, j}$ symmetric modes and the bunch width $\sigma_{x}=$ $500 \mu \mathrm{m}, \sigma_{y}=0$.

A $\mathrm{THz}$ structure was recently tested at Brookhaven National Laboratory [14], consisting of a rectangular waveguide loaded with polycrystalline chemical vapor deposition diamond plates as an accelerating structure. In this experiment, a drive (high charge) electron beam was launched through the structure exciting a wakefield following behind it. A smaller charge witness beam was launched at a variable delay with respect to the drive beam and experienced acceleration or deceleration depending on the delay. This measurement effectively maps the wakefield produced by the drive beam. The work presented

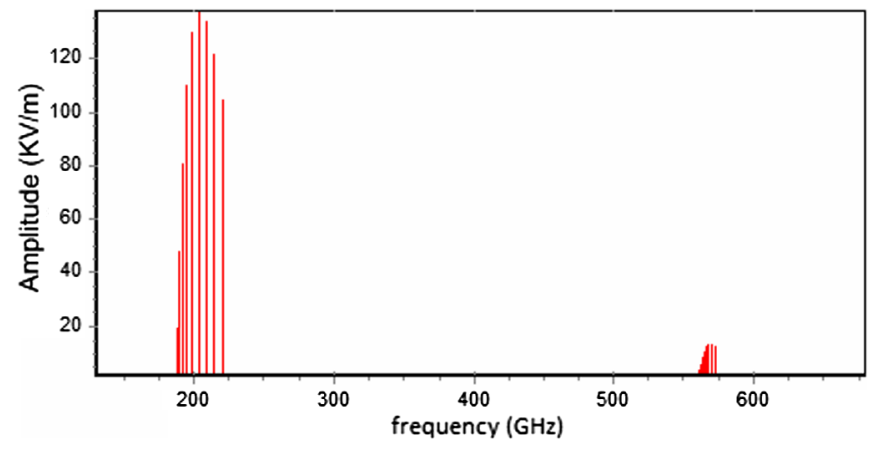

FIG. 5. $E_{z}$ field spectrum of the $L S E_{i, j}$ modes for $\sigma_{x}=$ $500 \mu \mathrm{m}, \sigma_{y}=0$. Bar group near the $L S E$ mode frequencies corresponds to higher $k_{x}$ values. 


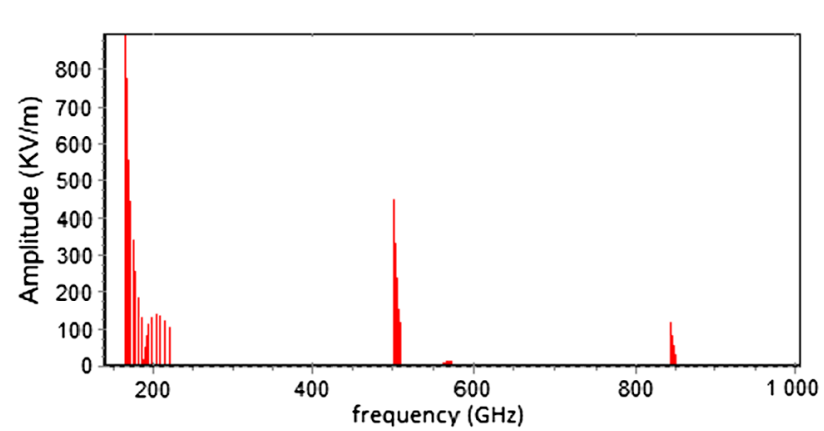

(a)

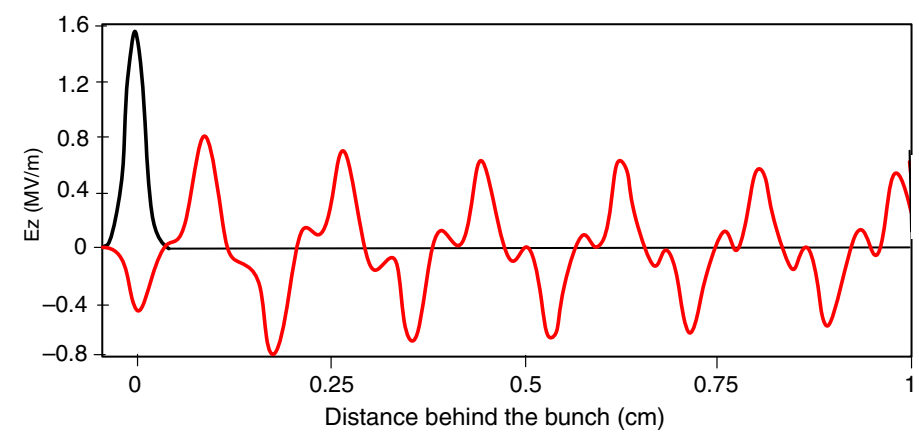

(b)

FIG. 6. (a) $E_{z}$ field spectrum (a) and $E_{z}$ field magnitude behind the bunch (b) for the bunch width $\sigma_{x}=500 \mu \mathrm{m}$.

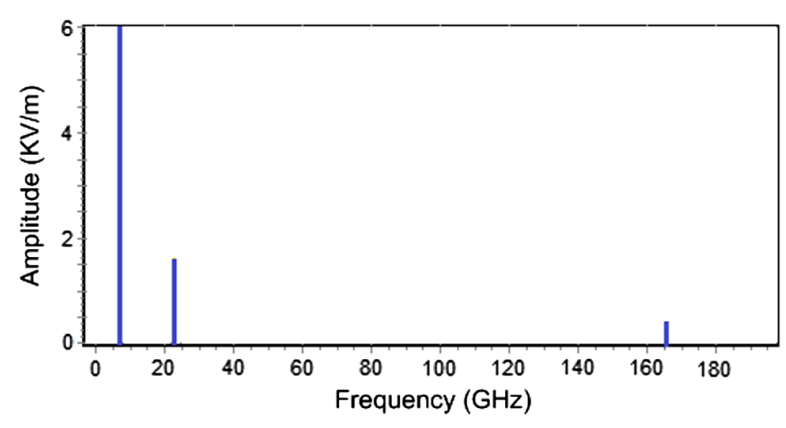

(a)

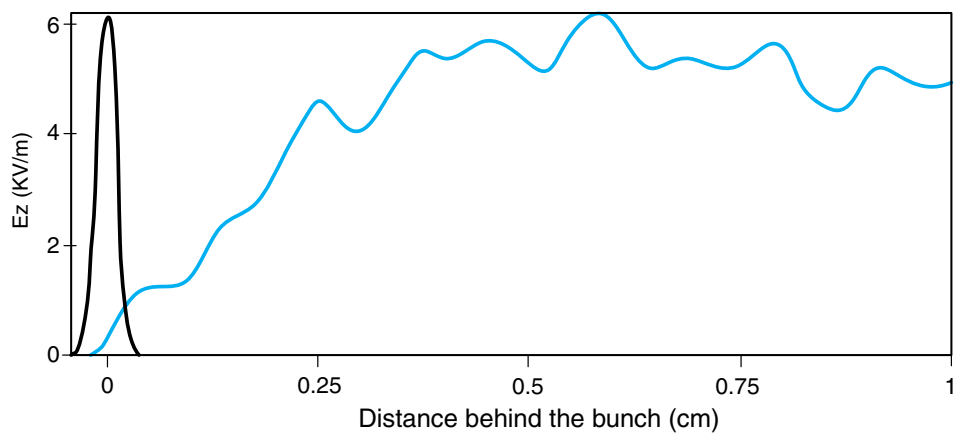

(b)

FIG. 7. $\quad F_{y}$ field spectrum (left) and distribution behind the bunch (right) for $40 \mu \mathrm{m}$ offset. Only $L S M_{1,1}$ and $L S M_{1,2}$ symmetric modes survive for $\sigma_{x}=5 \mathrm{~mm}, \sigma_{y}=0$. It is worth mentioning that this class of modes has an extremely low base frequency ( $7.6 \mathrm{GHz}$ ) and that their amplitude is also small.

in [14] represents the first mapping of the wakefield in a dielectric device in the $\mathrm{THz}$ regime (Table I).

The structure parameters presented in [14] and Table I provide a good opportunity to use the rigorous solution presented above for analytical simulations of the spectrum and magnitude of the wakefields excited by the bunch passing through a dual-layer rectangular DLA structure. $75 \mu \mathrm{m}$ thick polycrystalline diamond plates were loaded in a $6 \mathrm{~cm}$ long waveguide. The second outer layer with a thickness of $55-75 \mu \mathrm{m}$ between each diamond plate and conducting wall of the waveguide was formed by a dielectric glue with permittivity $\varepsilon \sim 1.8$. The beam gap was $2 b=200 \mu \mathrm{m}$. This structure yields a wakefield dominated by a $L S M_{11}$ mode with a $1200 \mu \mathrm{m}$ wavelength.

The ATF drive beam is short enough to excite higher order modes, hence the wake is not a pure sine wave. Parameter optimization carried out to fit the experimental data and analytical simulations with the formalism presented above allows us to predict the following parameters for the data fit of the experiment [14]: $b=100 \mu \mathrm{m}$,

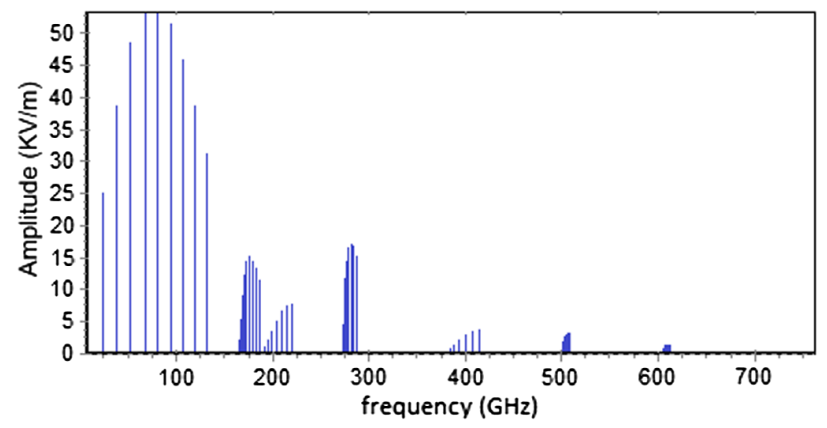

(a)

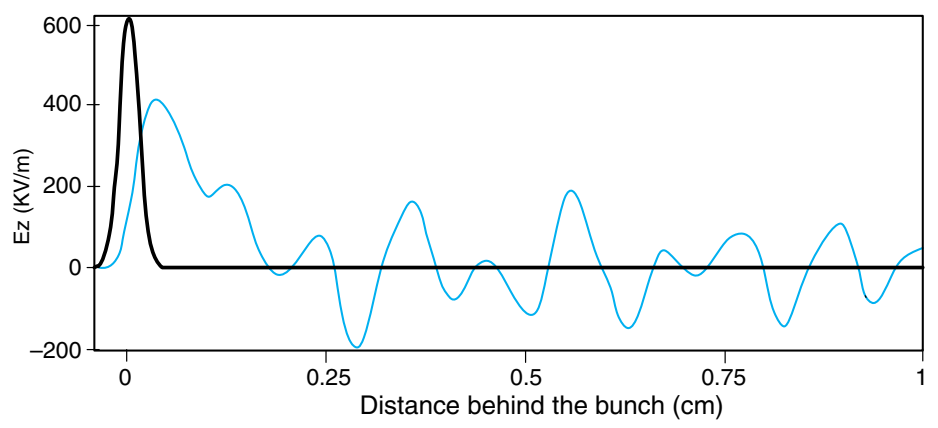

(b)

FIG. 8. $\quad F_{y}$ field spectrum (left) and distribution behind the bunch (right) for a $40 \mu \mathrm{m}$ beam offset. $L S M_{1, j}$ symmetric modes dominate for $\sigma_{x}=500 \mu \mathrm{m}, \sigma_{y}=0$. 


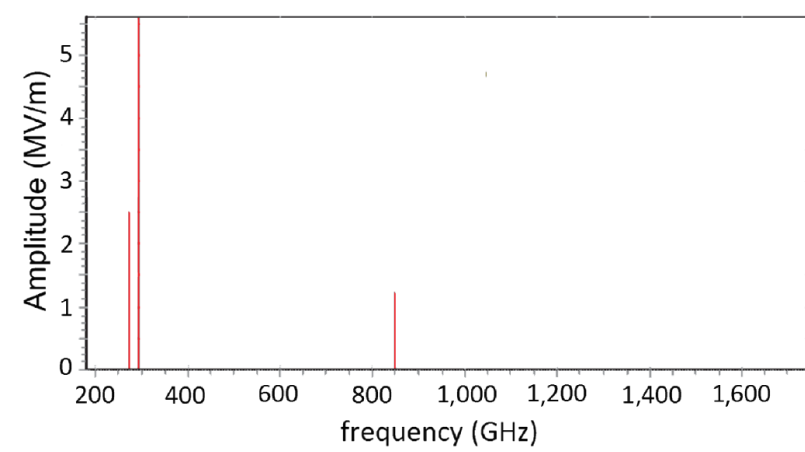

(a)

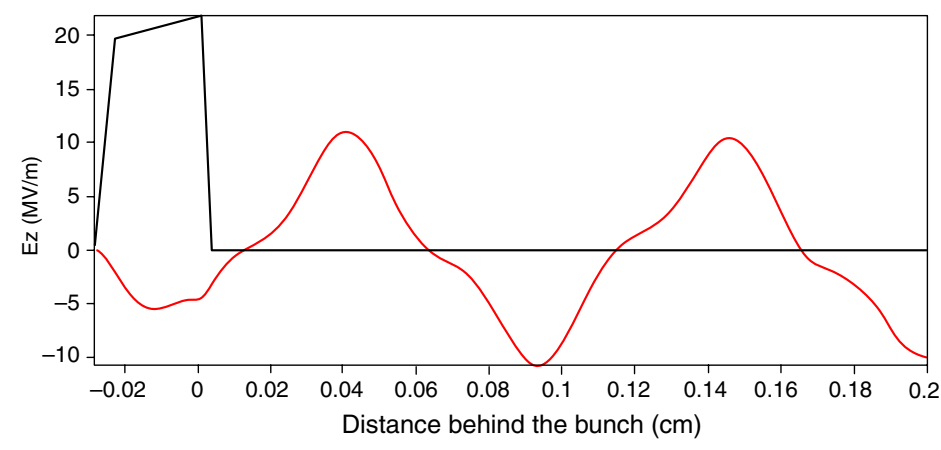

(b)

FIG. 9. (a) LSM mode spectrum for the structure parameters in [14] and a Gaussian electron bunch with $\sigma_{z} \sim 100 \mu \mathrm{m}$ and beam charge $70 \mathrm{pC}$; (b) the magnitude of the accelerating field $E_{z}$ for the structure parameters and the flattop beam charge distribution, $Q=70 \mathrm{pC}$ and length $320 \mu \mathrm{m}$, Ref. [14] and Table I.

$c=250 \mu \mathrm{m}$ consisting of a $75 \mu \mathrm{m}$ thick diamond plate and $\sim 75 \mu \mathrm{m}$ thick dielectric glue layer. Permittivity of the diamond slab is $\varepsilon_{1} \sim 5.7$, and for the dielectric glue layer $\varepsilon_{2}=1.8$. Figure 9(a) shows $L S M / L S E$ mode spectrum for the structure parameters presented above and a Gaussian electron beam with $\sigma_{z} \sim 100 \mu \mathrm{m}$ and beam charge $70 \mathrm{pC}$. The lowest frequency corresponds to an $L S E_{11}$ symmetric mode at $273 \mathrm{GHz}$ while the modes at 295 and $845 \mathrm{GHz}$ are $L S M_{11}$ and $L S M_{12}$ asymmetric modes, respectively. Figure 9(b) presents the magnitude of the accelerating field $E_{z}$ for the structure parameters, a flattop beam charge distribution $Q=70 \mathrm{pC}$ and length $320 \mu \mathrm{m}$ as it was used in the wakefield mapping experiment [14].

\section{CONCLUSION}

We have developed a modified method for the decomposition of Maxwell's equations by transverse operator eigenfunctions and we have applied this method to calculating the Cherenkov radiation fields in a rectangular waveguide. Our technique allows a rigorous full solution of the electromagnetic wakefields generated by a relativistic bunch moving along a rectangular dielectric-lined waveguide with nearly arbitrary dependence of the permittivity on the transverse coordinates.

A method based on the first order transverse operator has been previously applied to the analysis of Cherenkov radiation in waveguides, but at the same time the application of this method to transversely inhomogeneous rectangular waveguides is not obvious because of the problems of orthogonality between the components of the electric and magnetic field vectors and the definition of the bilinear form in $L_{2}$ space.

In this study we used a modification of the transverse operator method, where we considered the Sturm-Liouville second order operator with an alternating sign weight function. Consequently, the bi-orthogonality of the eigenfunctions as well as the similarity of the operator to a self-adjoint operator made it possible to obtain a rigorous and complete analytic solution for the eigenmodes and to solve in the most general form the problem of Cherenkov wakefield generation in a rectangular accelerating structure with a composite dielectric loading. This formalism does not require any assumptions on the self-Coulomb field of the beam, and we did not use the mode decomposition for the problem formulation and its subsequent solution. Finally, using the transverse operator method, the modes series for the solution is uniformly convergent.

The formalism described here was successfully applied to the analysis of rectangular dielectric-lined structures that were recently beam tested in experiments at the Argonne (ANL/AWA) and Brookhaven (BNL/ATF) accelerator facilities.

The analytic solutions obtained here were implemented in the RECTANGULAR code. The use of an analytic solutions based code makes it independent of the frequency range and the geometrical size of the structure. This ensures fast and effective analysis compared to the use of other standard numerical codes in the frequency range from $10 \mathrm{GHz}$ to up $1 \mathrm{THz}$, which usually require massive time consuming computations. In the Ka band frequency range, we have carried out analytical simulations of wakefields excited in rectangular dielectric accelerating structures; using this method for the Argonne/AWA beam parameters, we have analyzed a dielectric structure with a rectangular cross section, in which accelerating gradients higher than $100 \mathrm{MV} / \mathrm{m}$ can be attained. In the THz range, the structures tested at the BNL/ATF accelerator were considered and compared to experimental results.

\section{ACKNOWLEDGMENTS}

This work was supported by the Russian Foundation for Basic Research and the Ministry of Education and Science of the Russian Federation in the framework of the Federal Program "Human Capital for Science and Education in Innovative Russia" for 2009-2013. We give special thanks to P. Schoessow for useful discussions and suggestions. 


\section{APPENDIX}

In this Appendix we demonstrate the main steps of the proof that eigenfunctions of $\hat{T}_{E}$ and $\hat{T}_{H}$ operators form Riesz basis sets in $L_{2}$ space.

First of all, because of the symmetry of the system, the analysis of the $\hat{T}_{E}$ and $\hat{T}_{H}$ operator properties can be reduced to a simpler task. We consider operators expanded in a Fourier series in the $x$ coordinate. This reduces the analysis task from two dimensions (in the $X Y$ plane) to one dimension (only on the $y$ axis) with the additional parameter $k_{x}$. Further, the symmetry with respect to the origin of the $y$ axis gives us the opportunity to consider the reduced task on the interval $[0, c]$ only so that symmetric functions of $\hat{T}_{E}$ and $\hat{T}_{H}$ will satisfy the Neumann condition at 0 and the corresponding asymmetric functions to the Dirichlet condition at 0 . After rescaling the interval, it can be seen that the properties of the $\hat{T}_{E}$ and $\hat{T}_{H}$ operators are the same as the properties of the operator

$$
\hat{A}=-\rho\left[\frac{d^{2}}{d y^{2}}-C\right]
$$

where $\rho=1$ for $y \in[-1,0]$ and $\rho=-\kappa$ for $y \in(0,1]$, with the additional conditions on its domain $f(-0)=$ $\alpha f(+0), f^{\prime}(-0)=\beta f^{\prime}(+0)$. At the points \pm 1 for reconstructing all parts of the $\hat{T}_{E}$ and $\hat{T}_{H}$ operators we need to consider all combinations of conditions: DirichletDirichlet, Neumann-Neumann, etc. However, this does not affect the basis properties of eigenfunctions in principle. For simplicity of calculation we consider here the Dirichlet-Dirichlet conditions at the end of the interval. Here we assume that $\kappa, \alpha, \beta, C \in \mathbb{R}, C>0$ (although this method of proof will work for complex parameters also).

The equivalent eigenvalue problem can be formulated as follows:

$$
\begin{aligned}
-\rho\left[\frac{d^{2} f}{d y^{2}}-C f\right] & =\lambda f, \quad f(-0)=\alpha f(+0), \\
f^{\prime}(-0) & =\beta f^{\prime}(+0), \quad f(-1)=f(1)=0 .
\end{aligned}
$$

The proof is divided into four steps.

(i) Basic analysis and asymptotes.-One can check by direct calculation using root vectors formulas that all of them are equal to zero. Thus, the problem (A2) does not have any additional solutions except eigenfunctions that correspond to eigenvalues. Proceeding to the limit of large eigenvalues, one can determine the asymptotic eigenvalues as

$$
\begin{aligned}
& \lambda^{+}=\left(b_{1}+\pi n\right)^{2}+C, \\
& \lambda^{-}=-\kappa\left(-b_{1}+\frac{\pi}{2}+\pi n\right)^{2}-C .
\end{aligned}
$$

Here $b_{1}$ is given by

$$
b_{1}=\left[\frac{1-i \frac{\alpha \kappa}{\beta}}{1+i \frac{\alpha \kappa}{\beta}}\right] .
$$

One can see that spectrum is purely real but not positive. It consists of two branches. The first is purely positive and the second is purely negative.

(ii) Partial Gram matrix.-The Gram matrix is necessary to demonstrate the linear independence of the eigenfunctions inside each branch. Using the asymptotic eigenfunctions, one can build Gram matrices for each branch of eigenvalues. These matrices are equivalent and only one of them needs to be analyzed. We use the theorem that if the Gram matrix is invertible then vectors it is built from are linearly independent. A matrix that is similar to the Gram matrix of one branch has the form

$$
G_{n, m}=T_{n, m}-c_{1} H_{n, m}+\sigma_{2},
$$

where $G_{n, m}$ is the element of the Gram matrix, $T_{n, m}$ is the element of the Toeplitz matrix, $H_{n, m}$ is the element of the Hilbert matrix;

$$
T_{n, m}=\frac{1}{n-m}, \quad H_{n, m}=\frac{1}{n+m},
$$

and $\sigma_{2}$ is a Hilbert-Schmidt (compact) add-on, which can be ignored, $c_{1}$ is the constant that depends on initial parameters.

Hilbert and Toeplitz matrices are noncompact matrices. The matrix is invertible if its spectrum does not include zero. Using the fact that the Gram matrix is a nonnegative matrix in finite-dimensional space and then proceeding to the limit of an infinite dimensional space, one can show that

$$
G \geq a T,
$$

where $a>0$ is a constant, and $T$ is the Toelplitz matrix. Using representation of Toeplitz matrix as an operator of multiplication by a function on Hardy space, one can show that $T>0$. This implies the linear independence of the eigenfunctions inside one branch or in other words that each branch is a Riesz basis in its linear envelope.

(iii) Angle between eigensubspaces.-The two branches are linearly independent if the angle between eigensubspaces is nonzero (i.e. the subspaces do not coincide) in the limit $n, m \rightarrow \infty$ ( $n$ and $m$ are the indices of the first and the second branch). For the proof we use the technique of the Riesz projector and the fact that the Riesz projector is a parallel projector. We consider the Riesz projector $\hat{P}$ on one eigensubspace calculated over the contour $\Gamma_{1}$ which surrounds only one branch. The inverse relation of the projector's norm bounds the sine of the angle between the eigensubspaces:

$$
|\sin \phi| \leq \frac{1}{\|\hat{P}\|}
$$


Thus, to ensure that the angle is not equal to zero we should estimate the norm of this projector. This is done by using an additional self-adjoint resolvent of some operator $\hat{B}$ and considering the difference $\hat{P}_{d}$ between the resolvent of operator $\hat{A}$ and $\hat{B}$. Estimating the norm of

$$
\hat{P}_{d}=\int_{\Gamma_{1}} d \lambda\left(\frac{1}{I-\lambda \hat{A}}-\frac{1}{I-\lambda \hat{B}}\right)
$$

one can show that $\left\|\hat{P}_{d}\right\|<\infty$, which leads to the boundedness of the projector and nonzero angle $\phi$.

(iv) Completeness.-For completeness we need to show that the Riesz integral over the infinite contour $\Gamma_{2}$ which surrounds both branches is equal to the identity operator. This can again be done using as a supportive construction a self-adjoint resolvent and considering only the difference between the resolvent of operator $\hat{A}$ and a self-adjoint operator $\hat{B}$. In this case the Riesz integral of the difference should vanish at the infinity of the contour. This check can be made on any dense set of functions, for example on functions that are finite at $y=0$.

In (iii) and (iv) we suggest using the self-adjoint operator $\hat{B}$ given by

$$
\begin{aligned}
-\rho\left[\frac{d^{2} f}{d y^{2}}-C f\right] & =\lambda f, \quad f(-0)=\alpha f(+0), \\
f^{\prime}(-0) & =-1 / \alpha f^{\prime}(+0), \quad f(-1)=f(1)=0 .
\end{aligned}
$$

The results of the steps (i)-(iv) prove the statement that eigenfunctions of the problem (A2) form a Riesz basis in $L_{2}$.

[1] W. Leemans, B. Nagler, A. J. Gonsalves, Cs. Tóth, K. Nakamura, C. G. R. Geddes, E. Esarey, C. B. Schroeder, and S. M. Hooker, Nat. Phys. 2, 699 (2006).

[2] C. Joshi and V. Malka, New J. Phys. 12, 045003 (2010).

[3] Ian Blumenfeld, Christopher E. Clayton, Franz-Josef Decker, Mark J. Hogan, Chengkun Huang, Rasmus Ischebeck, Richard Iverson, Chandrashekhar Joshi, Thomas Katsouleas, Neil Kirby, Wei Lu, Kenneth A. Marsh, Warren B. Mori, Patric Muggli, Erdem Oz, Robert H. Siemann, Dieter Walz, and Miaomiao Zhou, Nature (London) 445, 7129 (2007).

[4] W. Gai, P. Schoessow, B. Cole, R. Konecny, J. Norem, J. Rosenzweig, and J. Simpson, Phys. Rev. Lett. 61, 2756 (1988).

[5] W. Gai, AIP Conf. Proc. 1086, 3 (2009).

[6] M. C. Thompson et al., Phys. Rev. Lett. 100, 214801 (2008).

[7] A. D. Kanareykin, J. Phys. Conf. Ser. 236, 012032 (2010).
[8] C.-J. Jing, S. P. Antipov, A. Kanareykin, P. Schoessow, M. E. Conde, W. Gai, and J. G. Power, in Proceedings of the 2011 Particle Accelerator Conference, NY, USA (IEEE, New York, 2011), p. 2279 [http:// accelconf.web.cern.ch/AccelConf/PAC2011/papers/thp078. pdf].

[9] C. Jing, A. Kanareykin, J. G. Power, and A. Zholents, in Proceedings of the 2nd International Particle Accelerator Conference, San Sebastián, Spain (EPS-AG, Spain, 2011), p. 1485 [http://dx.doi.org/10.1063/1.4790432].

[10] R. Tomás, Phys. Rev. ST Accel. Beams 13, 014801 (2010).

[11] C. Jing, S. P. Antipov, A. Kanareykin, P. Schoessow, M. E. Conde, W. Gai, J.G. Power, and I. Syratchev, in Proceedings of the 2011 Particle Accelerator Conference, NY, USA (Ref. [8]), p. 315 [http://accelconf.web.cern.ch/ AccelConf/PAC2011/papers/mop116.pdf].

[12] M. Conde, S. Antipov, W. Gai, and R. Konecny, in Proceedings of the International Particle Accelerator Conference, Kyoto, Japan (ICR, Kyoto, 2010), p. 4425 [http://accelconf.web.cern.ch/AccelConf/IPAC10/papers/ thpd062.pdf].

[13] J. Rosenzweig, G. Travish, M. Hogan, and P. Muggli, in Proceedings of the International Particle Accelerator Conference, Kyoto, Japan (Ref. [12]), p. 3605 [http:// accelconf.web.cern.ch/AccelConf/IPAC10/papers/ thoamh02.pdf].

[14] S. Antipov, C. Jing, A. Kanareykin, J. E. Butler, V. Yakimenko, M. Fedurin, K. Kusche, and W. Gai, Appl. Phys. Lett. 100, 132910 (2012).

[15] A. Altmark, A. Kanareykin, and I. Sheinman, Tech. Phys. 50, 87 (2005).

[16] C. Jing, A. Kanareykin, J. G. Power, M. Conde, W. Liu, S. Antipov, P. Schoessow, and W. Gai, Phys. Rev. Lett. 106, 164802 (2011).

[17] A. Kanareykin, AIP Conf. Proc. 1299, 286 (2010).

[18] A. Kanareykin, P. Schoessow, M. Conde, C. Jing, J. G. Power, and W. Gai, in Proceedings of the 10th European Particle Accelerator Conference, Edinburgh, Scotland, 2006 (EPS-AG, Edinburgh, Scotland, 2006), p. 2460.

[19] C. Wang, V.P. Yakovlev, M.A. LaPointe, and J. L. Hirshfield, in Proceedings of the 10th European Particle Accelerator Conference, Edinburgh, Scotland, 2006 (Ref. [18]), p. 2457.

[20] B. M. Bolotvskii, Usp. Fiz. Nauk 75, 295 (1961) [Sov. Phys. Usp. 4, 781 (1962)].

[21] M. Rosing and W. Gai, Phys. Rev. D 42, 1829 (1990).

[22] S. Antipov, C. Jing, P. Schoessow, A. Kanareykin, W. Gai, A. Zholents, M. Fedurin, K. Kusche, and V. Yakimenko, in Proceedings of the 3rd International Particle Accelerator Conference, New Orleans, Louisiana, USA, 2012 (Ref. [38]), p. 598.

[23] A. Tremaine, J. Rosenzweig, and P. Schoessow, Phys. Rev. E 56, 7204 (1997).

[24] P. Piot, Y.-E Sun, and K.-J. Kim, Phys. Rev. ST Accel. Beams 9, 031001 (2006).

[25] L. Xiao, W. Gai, and X. Sun, Phys. Rev. E 65, 016505 (2001).

[26] C. Jing, W. Liu, W. Gai, L. Xiao, and T. Wong, Phys. Rev. E 68, 016502 (2003). 
[27] A. D. Kanareikin and I. L. Sheinman, Tech. Phys. Lett. 33, 344 (2007).

[28] S. Y. Park, C. Wang, and J. L. Hirshfield, AIP Conf. Proc. 647, 527 (2002).

[29] C. Wang and J. L. Hirshfield, Phys. Rev. ST Accel. Beams 9, 031301 (2006).

[30] G. V. Sotnikov, I. N. Onishchenko, J. L. Hirshfield, and T. C. Marshall, Probl. At. Nauki Tekhnol. Ser.: Yad._Fiz. Issled. 49, 148 (2008).

[31] D. Bresler, G. H. Joshi, and N. Marcuvitz, J. Appl. Phys. 29, 794 (1958).

[32] A. Rowland and J. Sammut, J. Opt. Soc. Am. 72, 1335 (1982).

[33] J.W. Tao, J. Atechian, P. Ratovondrahanta, and H. Baudrand, Proc. IEEE 137, 311 (1990).

[34] C. A. Balanis, Advanced Engineering Electromagnetics (Wiley, New York, 1989), pp. 410-414.

[35] O. A. Tretyakov, Analytical and Numerical Methods in Electromagnetic Wave Theory, edited by M. Hashimoto, M. Idemen, and O. A. Tretyakov (Science House Co., Ltd., Tokyo, 1993), p. 572.
[36] A. Y. Butrym and B.A. Kochetov, Progress in Electromagn. Res. B 19, 151 (2010).

[37] http://www.cst.com.

[38] S. Antipov, C. Jing, P. Schoessow, J. E. Butler, S. Zuo, A. Kanareykin, M. Conde, D.S. Doran, W. Gai, R.S. Konecny, J. G. Power, Z. Yusof, and S. Baryshev, in Proceedings of the 3rd International Particle Accelerator Conference, New Orleans, Louisiana, USA, 2012 (IEEE, Piscataway, NJ, 2012), p. 2813 [http://accelconf.web.cern.ch/AccelConf/IPAC2012/papers/ weppp041.pdf].

[39] A. Kanareykin, S.P. Antipov, J.E. Butler, C.-J. Jing, and S.S. Zuo, in Proceedings of the 3rd International Particle Accelerator Conference, New Orleans, Louisiana, USA, 2012 (Ref. [38]), p. 2819 [http://accelconf.web.cern.ch/accelconf/IPAC2012/papers/ WEPPP041.pdf].

[40] G. Andonian, D. Stratakis, M. Babzien, S. Barber, M. Fedurin, E. Hemsing, K. Kusche, P. Muggli, B. O’Shea, X. Wei, O. Williams, V. Yakimenko, and J.B. Rosenzweig, Phys. Rev. Lett. 108, 244801 (2012). 